\title{
Correlators in the Gaussian and chiral supereigenvalue models in the Neveu-Schwarz sector
}

\author{
Rui Wang, ${ }^{a}$ Shi-Kun Wang, ${ }^{a}$ Ke Wu ${ }^{b}$ and Wei-Zhong Zhao ${ }^{b, 1}$ \\ ${ }^{a}$ Institute of Applied Mathematics, Academy of Mathematics and Systems Science, \\ Chinese Academy of Sciences, \\ Beijing 100190, China \\ ${ }^{b}$ School of Mathematical Sciences, Capital Normal University, \\ Beijing 100048, China \\ E-mail: wrui@amss.ac.cn, wsk@amss.ac.cn, wuke@cnu.edu.cn, \\ zhaowz@cnu.edu.cn
}

ABstract: We analyze the Gaussian and chiral supereigenvalue models in the NeveuSchwarz sector. We show that their partition functions can be expressed as the infinite sums of the homogeneous operators acting on the elementary functions. In spite of the fact that the usual $W$-representations of these matrix models can not be provided here, we can still derive the compact expressions of the correlators in these two supereigenvalue models. Furthermore, the non-Gaussian (chiral) cases are also discussed.

KEYwords: Conformal and W Symmetry, Matrix Models

ArXiv EPrint: 2009.02929

\footnotetext{
${ }^{1}$ Corresponding author.
} 


\section{Contents}

1 Introduction 1

2 Gaussian supereigenvalue model in the Neveu-Schwarz sector 2

3 Non-Gaussian supereigenvalue model in the Neveu-Schwarz sector $\quad 10$

4 Chiral supereigenvalue model in the Neveu-Schwarz sector 13

5 Non-Gaussian chiral supereigenvalue model in the Neveu-Schwarz sector 16

6 Conclusions

\section{Introduction}

The supereigenvalue models have attracted considerable attention. They can be regarded as supersymmetric generalizations of matrix models [1]-[17]. Many of the important features of matrix models, such as the Virasoro constraints, the loop equations, the genus expansions and the moment descriptions have their supersymmetric counterparts in the supereigenvalue models. Two types of supereigenvalue models in the Ramond sector, i.e., Ramond-NS and Ramond-R supereigenvalue models, were derived in ref. [14] by means of the conformal field theory formalism. They obey the super Virasoro constraints, where the bosonic and fermionic operators yield the Ramond version of the super-Virasoro algebra. Moreover, the corresponding Ramond super quantum curves were analyzed and the underlying Ramond super spectral curves were identified. It is well-known that the $W$-representations can be used to realize partition functions of various matrix models, such as the Gaussian Hermitian and complex matrix models [18]-[21], the Kontsevich matrix model [22] and the generalized Brezin-Gross-Witten model [23]. Namely, by acting on elementary functions with exponents of the given $W$-operators, we can give the corresponding partition functions of the matrix models. Recently it was proved that Ramond-NS supereigenvalue model can be obtained in terms of the $W$-representation [24]

$$
\begin{aligned}
Z & =\left(\prod_{i=1}^{N} \int_{0}^{+\infty} d z_{i} \int d \theta_{i}\right) \Delta_{R}(z, \theta)^{\beta} e^{-\frac{\sqrt{\beta}}{\hbar} \sum_{i=1}^{N}\left(\sum_{k=0}^{\infty}\left(t_{k} z_{i}^{k}+\xi_{k} z_{i}^{k-\frac{1}{2}} \theta_{i}\right)+z_{i}\right)} \\
& =e^{-W} e^{-\frac{\sqrt{\beta}}{\hbar} N t_{0}}
\end{aligned}
$$

where $N$ is even, $z_{i}$ are bosonic variables and $\theta_{i}$ are Grassmann variables, $t_{k}$ and $\xi_{k}$ are bosonic and fermionic coupling constants, respectively, $\Delta_{R}(z, \theta)$ is the Vandermondelike determinant, $\Delta_{R}(z, \theta)=\prod_{1 \leq i<j \leq N}\left(z_{i}-z_{j}-\frac{1}{2}\left(z_{i}+z_{j}\right) \frac{\theta_{i} \theta_{j}}{\sqrt{z_{i} z_{j}}}\right)$. Due to the $W$ representation, the compact expressions of correlators in the supereigenvalue model (1.1) 
can be derived. The final result shows that the correlators are determined by the certain coefficients in the power of the operator $W$.

The supereigenvalue model in the Neveu-Schwarz sector describes the coupling between $(2,4 m)$ superconformal models and world-sheet supergravity [1]. The $\beta$-deformed case of this supereigenvalue model is given by [12]

$$
\tilde{Z}=\left(\prod_{i=1}^{N} \int_{-\infty}^{+\infty} d z_{i} \int d \theta_{i}\right) \Delta_{N S}(z, \theta)^{\beta} e^{-\frac{\sqrt{\beta}}{\hbar} \sum_{i=1}^{N} V_{N S}\left(z_{i}, \theta_{i}\right)},
$$

where $N$ is even, $\Delta_{N S}(z, \theta)=\prod_{1 \leq i<j \leq N}\left(z_{i}-z_{j}-\theta_{i} \theta_{j}\right), V_{N S}(z, \theta)=\sum_{k=0}^{\infty} t_{k} z^{k}+$ $\sum_{k=0}^{\infty} \xi_{k+\frac{1}{2}} z^{k} \theta$, and $\xi_{k+\frac{1}{2}}$ are fermionic coupling constants. There are the super Virasoro constraints for the partition function (1.2), where the bosonic and fermionic operators yield the Neveu-Schwarz version of the super-Virasoro algebra. When the bosonic variables $z_{i}$ in (1.2) are integrated from 0 to $+\infty$, it gives the chiral supereigenvalue model, which can be seen as the supersymmetric generalization of the complex one-matrix model. The closed expressions for all planar multi-superloop correlation functions were derived for $\beta=1$ chiral supereigenvalue model. It was found that the higher genus contributions to the free energy and the correlators can be calculated by an iterative scheme [10].

It is known that the compact expressions of the correlators in the supereigenvalue model (1.1) in the Ramond sector have been presented. However, for the cases of the supereigenvalue model in the Neveu-Schwarz sector, it still remains to be seen whether there are the similar results. In this paper, we analyze the (non-)Gaussian supereigenvalue model in the Neveu-Schwarz sector and give the correlators in these matrix models. Moreover, the cases of the chiral supereigenvalue model will be also investigated.

This paper is organized as follows. In section 2, we focus on the Gaussian supereigenvalue model in the Neveu-Schwarz sector and show that its partition function can be expressed as the infinite sums of the homogeneous operators acting on the elementary functions. Moreover, we derive the compact expressions of the correlators. In section 3, we consider the non-Gaussian supereigenvalue model in the Neveu-Schwarz sector. In section 4 , we consider the chiral supereigenvalue model and present the compact expressions of the correlators. The non-Gaussian chiral case is analyzed in section 5. We end this paper with the conclusions in section 6 .

\section{Gaussian supereigenvalue model in the Neveu-Schwarz sector}

The Gaussian Hermitian matrix model is one of the most studied and best understood matrix models. Its partition function can be generated by the $W$-representation [18]. The correlators in this matrix model have been well discussed [25]-[35] and the compact expressions of the correlators have been presented [33]-[35]. For the non-Gaussian Hermitian matrix model, the correlators can be evaluated by the recursive formulas derived from the Virasoro constraints and the additional constraints. However, it is hard to give the compact expressions of the correlators [36-40]. 
In this section, we focus on the Gaussian supereigenvalue model which is obtained by taking the shift $t_{2} \rightarrow t_{2}+\frac{1}{2}$ in the potential of the partition function (1.2)

$$
Z_{G}=\frac{1}{\Lambda_{G}}\left(\prod_{i=1}^{N} \int_{-\infty}^{+\infty} d z_{i} \int d \theta_{i}\right) \Delta_{N S}(z, \theta)^{\beta} e^{-\frac{\sqrt{\beta}}{\hbar} \sum_{i=1}^{N}\left(V_{N S}\left(z_{i}, \theta_{i}\right)+\frac{1}{2} z_{i}^{2}\right)},
$$

where the normalization factor $\Lambda_{G}$ is given by

$$
\Lambda_{G}=\left(\prod_{i=1}^{N} \int_{-\infty}^{+\infty} d z_{i} \int d \theta_{i}\right) \Delta_{N S}(z, \theta)^{\beta} e^{-\frac{\sqrt{\beta}}{2 \hbar} \sum_{i=1}^{N} z_{i}^{2}}
$$

From the invariance of the integral (2.1) under the changes of integration variables $\left(z_{i} \rightarrow z_{i}+\epsilon z_{i}^{n+1}, \quad \theta_{i} \rightarrow \theta_{i}+\frac{1}{2} \epsilon(n+1) \theta_{i} z_{i}^{n}\right)$ and $\left(z_{i} \rightarrow z_{i}+z_{i}^{n+1} \theta_{i} \delta, \quad \theta_{i} \rightarrow \theta_{i}+z_{i}^{n+1} \delta\right)$, where $\epsilon$ and $\delta$ are the infinitesimal bosonic and fermionic constants, respectively, we can give the super Virasoro constraints

$$
\ell_{n} Z_{G}=0, \quad g_{n+\frac{1}{2}} Z_{G}=0, \quad n \geq-1
$$

where

$$
\begin{aligned}
\ell_{n}= & \frac{\partial}{\partial t_{n+2}}+\sum_{k=1}^{\infty} k t_{k} \frac{\partial}{\partial t_{n+k}}+\frac{\hbar^{2}}{2} \sum_{k=0}^{n} \frac{\partial}{\partial t_{k}} \frac{\partial}{\partial t_{n-k}}+\sum_{k=0}^{\infty}\left(k+\frac{n+1}{2}\right) \xi_{k+\frac{1}{2}} \frac{\partial}{\partial \xi_{k+n+\frac{1}{2}}} \\
& +\frac{\hbar^{2}}{2} \sum_{k=1}^{n} k \frac{\partial}{\partial \xi_{n-k+\frac{1}{2}}} \frac{\partial}{\partial \xi_{k-\frac{1}{2}}}-\frac{\hbar}{2 \sqrt{\beta}}(1-\beta)(n+1) \frac{\partial}{\partial t_{n}} \\
g_{n+\frac{1}{2}}= & \frac{\partial}{\partial \xi_{n+\frac{5}{2}}}+\sum_{k=1}^{\infty} k t_{k} \frac{\partial}{\partial \xi_{n+k+\frac{1}{2}}}+\sum_{k=0}^{\infty} \xi_{k+\frac{1}{2}} \frac{\partial}{\partial t_{k+n+1}}+\hbar^{2} \sum_{k=0}^{n} \frac{\partial}{\partial \xi_{k+\frac{1}{2}}} \frac{\partial}{\partial t_{n-k}} \\
& -\frac{\hbar}{\sqrt{\beta}}(1-\beta)(n+1) \frac{\partial}{\partial \xi_{n+\frac{1}{2}}} .
\end{aligned}
$$

The constraint operators obey the Neveu-Schwarz version of the super-Virasoro algebra

$$
\begin{aligned}
{\left[\ell_{m}, \ell_{n}\right] } & =(m-n) \ell_{m+n}, \\
{\left[\ell_{m}, g_{n+\frac{1}{2}}\right] } & =\left(\frac{m-1}{2}-n\right) g_{m+n+\frac{1}{2}}, \\
\left\{g_{m+\frac{1}{2}}, g_{n+\frac{1}{2}}\right\} & =2 \ell_{m+n+1} .
\end{aligned}
$$

Let us now take the change of variables given by

$$
z_{i} \rightarrow z_{i}+\epsilon \sum_{n=1}^{\infty} n t_{n} z_{i}^{n-1}, \quad \theta_{i} \rightarrow \theta_{i}+\frac{1}{2} \epsilon \sum_{n=2}^{\infty} n(n-1) t_{n} z_{i}^{n-2} \theta_{i} .
$$

By requiring that the partition function is invariant under the infinitesimal transformations (2.6), it leads to the constraint

$$
\left(\tilde{D}_{1}+\tilde{W}_{1}\right) Z_{G}=0
$$


where

$$
\begin{aligned}
\tilde{D}_{1}= & \sum_{k=1}^{\infty} k t_{k} \frac{\partial}{\partial t_{k}}, \\
\tilde{W}_{1}= & \sum_{n, k=1}^{\infty} n k t_{n} t_{k} \frac{\partial}{\partial t_{n+k-2}}+\sum_{n=1}^{\infty} \sum_{k=0}^{\infty} n\left(k+\frac{n-1}{2}\right) t_{n} \xi_{k+\frac{1}{2}} \frac{\partial}{\partial \xi_{n+k-\frac{3}{2}}} \\
& +\frac{\hbar^{2}}{2} \sum_{n=2}^{\infty} \sum_{k=0}^{n-2} n t_{n} \frac{\partial}{\partial t_{k}} \frac{\partial}{\partial t_{n-k-2}}+\frac{\hbar^{2}}{2} \sum_{n=3}^{\infty} \sum_{k=1}^{n-2} n k t_{n} \frac{\partial}{\partial \xi_{n-k-\frac{3}{2}}} \frac{\partial}{\partial \xi_{k-\frac{1}{2}}} \\
& -\frac{\hbar}{2 \sqrt{\beta}}(1-\beta) \sum_{n=2}^{\infty} n(n-1) t_{n} \frac{\partial}{\partial t_{n-2}} .
\end{aligned}
$$

Similarly, by requiring the invariance of the partition function (2.1) under

$$
z_{i} \rightarrow z_{i}+\epsilon \sum_{n=1}^{\infty}\left(n+\frac{1}{2}\right) \xi_{n+\frac{1}{2}} z_{i}^{n-1} \theta_{i}, \quad \theta_{i} \rightarrow \theta_{i}-\epsilon \sum_{n=1}^{\infty}\left(n+\frac{1}{2}\right) \xi_{n+\frac{1}{2}} z_{i}^{n-1},
$$

it gives another constraint

$$
\left(\tilde{D}_{2}+\tilde{W}_{2}\right) Z_{G}=0
$$

where

$$
\begin{aligned}
\tilde{D}_{2}= & \sum_{k=1}^{\infty}\left(k+\frac{1}{2}\right) \xi_{k+\frac{1}{2}} \frac{\partial}{\partial \xi_{k+\frac{1}{2}}}, \\
\tilde{W}_{2}= & \sum_{n, k=1}^{\infty} n\left(k+\frac{1}{2}\right) t_{n} \xi_{k+\frac{1}{2}} \frac{\partial}{\partial \xi_{n+k-\frac{3}{2}}}-\frac{\hbar}{\sqrt{\beta}}(1-\beta) \sum_{n=2}^{\infty}(n-1)\left(n+\frac{1}{2}\right) \xi_{n+\frac{1}{2}} \frac{\partial}{\partial \xi_{n-\frac{3}{2}}} \\
& +\hbar^{2} \sum_{n=2}^{\infty} \sum_{k=0}^{n-2}\left(n+\frac{1}{2}\right) \xi_{n+\frac{1}{2}} \frac{\partial}{\partial \xi_{k+\frac{1}{2}}} \frac{\partial}{\partial t_{n-k-2}}+\sum_{n=1}^{\infty} \sum_{k=0}^{\infty}\left(n+\frac{1}{2}\right) \xi_{n+\frac{1}{2}} \xi_{k+\frac{1}{2}} \frac{\partial}{\partial t_{n+k-1}} .
\end{aligned}
$$

Combining (2.7) and (2.10), we have

$$
(\tilde{D}+\tilde{W}) Z_{G}=0
$$

where $\tilde{D}=\tilde{D}_{1}+\tilde{D}_{2}, \tilde{W}=\tilde{W}_{1}+\tilde{W}_{2}$.

The partition function (2.1) can be formally expanded as

$$
\begin{aligned}
Z_{G}= & e^{-\frac{\sqrt{\beta}}{\hbar} N t_{0}}+\sum_{s=1}^{\infty} Z_{G}^{(s)} \\
= & e^{-\frac{\sqrt{\beta}}{\hbar} N t_{0}}\left[1-\frac{\sqrt{\beta}}{\hbar} \tilde{C}_{k_{1}} t_{k_{1}}+\frac{1}{2 !}\left(\frac{\sqrt{\beta}}{\hbar}\right)^{2} \tilde{C}_{k_{1}, k_{2}} t_{k_{1}} t_{k_{2}}-\frac{1}{2 !}\left(\frac{\sqrt{\beta}}{\hbar}\right)^{2} \tilde{C}^{s_{1}+\frac{1}{2}, s_{2}+\frac{1}{2}} \xi_{s_{1}+\frac{1}{2}} \xi_{s_{2}+\frac{1}{2}}\right. \\
& \left.-\frac{1}{3 !}\left(\frac{\sqrt{\beta}}{\hbar}\right)^{3} \tilde{C}_{k_{1}, k_{2}, k_{3}} t_{k_{1}} t_{k_{2}} t_{k_{3}}+\frac{1}{2 !}\left(\frac{\sqrt{\beta}}{\hbar}\right)^{3} \tilde{C}_{k_{1}}^{s_{1}+\frac{1}{2}, s_{2}+\frac{1}{2}} t_{k_{1}} \xi_{s_{1}+\frac{1}{2}} \xi_{s_{2}+\frac{1}{2}}+\cdots\right], \quad(2.13)
\end{aligned}
$$


where

$$
\begin{aligned}
Z_{G}^{(s)}= & e^{-\frac{\sqrt{\beta}}{\hbar} N t_{0}}\left[\sum_{n=0}^{\infty} \sum_{\begin{array}{c}
m=0 \\
m \text { is even }
\end{array}}^{\infty} \sum_{\begin{array}{r}
k_{1}+\cdots+k_{n}+ \\
s_{1}+\cdots+s_{m}+\frac{m}{2}=s \\
k_{1}, \cdots, k_{n} \geq 1 \\
s_{1}, \cdots, s_{m} \geq 0
\end{array}} \frac{(-1)^{\frac{m(m+1)}{2}}\left(-\frac{\sqrt{\beta}}{\hbar}\right)^{n+m}}{n ! m !} \tilde{C}_{k_{1}, \cdots, k_{n}}^{s_{1}+\frac{1}{2}, \cdots, s_{m}+\frac{1}{2}}\right. \\
& \left.\cdot t_{k_{1}} \cdots t_{k_{n}} \xi_{s_{1}+\frac{1}{2}} \cdots \xi_{s_{m}+\frac{1}{2}}\right]
\end{aligned}
$$

and the coefficients $\tilde{C}_{k_{1}, \cdots, k_{n}}^{s_{1}+\frac{1}{2}, \cdots, s_{m}+\frac{1}{2}}$ are the correlators defined by

$$
\begin{aligned}
\tilde{C}_{k_{1}, \cdots, k_{n}}^{s_{1}+\frac{1}{2}, \cdots, s_{m}+\frac{1}{2}}= & \frac{1}{\Lambda_{G}}\left(\prod_{i=1}^{N} \int_{-\infty}^{+\infty} d z_{i} \int d \theta_{i}\right) \sum_{\substack{i_{1}, \cdots, i_{n}=1 \\
j_{1}, \cdots, j_{m}=1}}^{N} z_{i_{1}}^{k_{1}} \cdots z_{i_{n}}^{k_{n}} z_{j_{1}}^{s_{1}} \theta_{j_{1}} \cdots z_{j_{m}}^{s_{m}} \theta_{j_{m}} \\
& \cdot \Delta_{N S}(z, \theta)^{\beta} e^{-\frac{\sqrt{\beta}}{2 \hbar} \sum_{i=1}^{N} z_{i}^{2}} .
\end{aligned}
$$

Let $V$ be the infinite dimensional vector space on $\mathbb{C}\left[\left[t_{0}\right]\right]$ generated by the basis

$$
\left\{t_{k_{1}} \cdots t_{k_{n}} \xi_{s_{1}+\frac{1}{2}} \cdots \xi_{s_{m}+\frac{1}{2}} \mid k_{1}, \cdots, k_{n} \geq 1, s_{1}, \cdots, s_{m} \geq 0, n \in \mathbb{N}, m \in 2 \mathbb{N}\right\}
$$

Thus the partition function $Z_{G}$ can be seen as a vector in $V$, the operators $\tilde{D}$ and $\tilde{W}$ are differential operators on $V$. Under the natural gradation $\operatorname{deg}\left(t_{k}\right)=k, \operatorname{deg}\left(\frac{\partial}{\partial t_{k}}\right)=-k$, $\operatorname{deg}\left(\xi_{k+\frac{1}{2}}\right)=k+\frac{1}{2}$, and $\operatorname{deg}\left(\frac{\partial}{\partial \xi_{k+\frac{1}{2}}}\right)=-k-\frac{1}{2}, k=0,1, \cdots, \infty$, we have $\operatorname{deg}(\tilde{D})=0$ and $\operatorname{deg}(\tilde{W})=2$. In addition, the kernel of $\tilde{D}$ denoted as $\operatorname{Ker}(\tilde{D})=\{v \in V \mid \tilde{D} v=0\}$, is one dimensional. It is not difficult to see that $\operatorname{Ker}(\tilde{D}+\tilde{W})$ is still one dimensional. Therefore, the partition function $Z_{G}$ is uniquely determined by the constraint (2.12). As a consequence, the correlators (2.15) can be totally derived from (2.12).

Let us collect the coefficients of $t_{1}^{l}$ and $t_{2}^{l}$ in (2.12) and set to zero, respectively, we obtain

$$
\tilde{C}_{1}=0, \quad \tilde{C}_{1,1}=\frac{\hbar}{\sqrt{\beta}} N, \quad \tilde{C}_{2}=\frac{\hbar}{2 \sqrt{\beta}} N \tilde{N}
$$

and the recursive relations

$$
\begin{aligned}
& \tilde{C}_{\underbrace{1, \cdots, 1}_{l}}=\frac{\hbar}{\sqrt{\beta}} N(l-1) \tilde{C}_{1, \cdots, 1}, \\
& \tilde{C}_{l-2}^{\tilde{C}_{l}, \cdots, 2}=\frac{\hbar}{\sqrt{\beta}}\left[\frac{N \tilde{N}}{2}+2(l-1)\right] \tilde{C}_{\underbrace{2, \cdots, 2}_{l-1}},
\end{aligned}
$$

where $\tilde{N}=\beta N+1-\beta$.

Then it is easy to obtain

$$
\tilde{C}_{l}^{\tilde{C}_{1, \cdots, 1}}= \begin{cases}\left(\frac{\hbar}{\sqrt{\beta}} N\right)^{\frac{l}{2}}(l-1) ! !, & \text { for } l \text { even; } \\ 0, & \text { for } l \text { odd }\end{cases}
$$


and

$$
\tilde{C}_{\underbrace{2, \cdots, 2}_{l}}=\left(\frac{\hbar}{\sqrt{\beta}}\right)^{l} \prod_{j=0}^{l-1}\left(\frac{N \tilde{N}}{2}+2 j\right) .
$$

Similarly, by collecting the coefficients of $t_{1}^{l} t_{2}, t_{1}^{l} \xi_{\frac{1}{2}} \xi_{\frac{3}{2}}, t_{2}^{l} t_{1}^{2}$ and $t_{2}^{l} \xi_{\frac{1}{2}} \xi_{\frac{3}{2}}$ in (2.12) and setting to zero, respectively, we may also obtain the corresponding recursive relations and derive the exact correlators

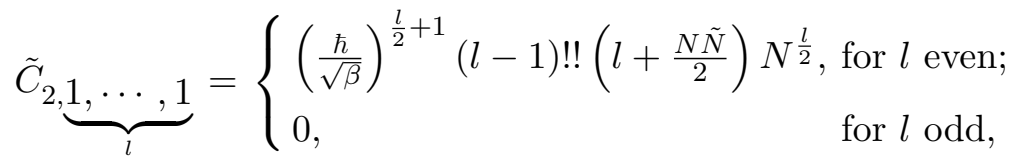

$$
\begin{aligned}
& \underbrace{\tilde{C}_{1}^{\frac{1}{2}, \frac{3}{2}}, \cdots, 1}_{l}= \begin{cases}\left(\frac{\hbar}{\sqrt{\beta}} N\right)^{\frac{l}{2}+1}(l-1) ! !, & \text { for } l \text { even; } \\
0, & \text { for } l \text { odd },\end{cases} \\
& \tilde{C}_{1,1, \underbrace{2, \cdots, 2}_{l}}=\underbrace{\tilde{C}_{2}^{\frac{1}{2}, \frac{3}{2}}, \cdots, 2}_{l}=\left(\frac{\hbar}{\sqrt{\beta}}\right)^{l+1} N \prod_{j=1}^{l}\left(\frac{N \tilde{N}}{2}+2 j\right) \text {. }
\end{aligned}
$$

It should be mentioned that we can also calculate the correlators (2.19)-(2.21) from the super Virasoro constraints (2.3). However, due to the complicated form of the recursive formulas for correlators from (2.3), it is hard to give the general expressions of the correlators. In order to achieve more results, let us further analyze the constraint (2.12).

Since $\tilde{D} e^{-\frac{\sqrt{\beta}}{\hbar} N t_{0}}=0$, the constraint $(2.12)$ can be rewritten as

$$
(\tilde{D}+\tilde{W}) \sum_{s=1}^{\infty} Z_{G}^{(s)}=-\tilde{W} e^{-\frac{\sqrt{\beta}}{\hbar} N t_{0}} .
$$

It is noted that the function $-\tilde{W} e^{-\frac{\sqrt{\beta}}{\hbar} N t_{0}}$ on the right hand side of $(2.22)$ has degree 2. Hence the operators $\tilde{D}$ and $\tilde{D}+\tilde{W}$ are invertible on $-\tilde{W} e^{-\frac{\sqrt{\beta}}{\hbar} N t_{0}}$.

From (2.22), we have

$$
\begin{aligned}
\sum_{s=1}^{\infty} Z_{G}^{(s)} & =-(\tilde{D}+\tilde{W})^{-1} \tilde{W} e^{-\frac{\sqrt{\beta}}{\hbar} N t_{0}} \\
& =\sum_{k=1}^{\infty}\left(-\tilde{D}^{-1} \tilde{W}\right)^{k} e^{-\frac{\sqrt{\beta}}{\hbar} N t_{0}}
\end{aligned}
$$

Thus the partition function (2.1) can be expressed as

$$
Z_{G}=\sum_{k=0}^{\infty}\left(-\tilde{D}^{-1} \tilde{W}\right)^{k} e^{-\frac{\sqrt{\beta}}{\hbar} N t_{0}} .
$$

We denote an operator $\mathcal{O}$ on $V$ the degree operator if $\mathcal{O} f=\operatorname{deg}(f) f$ for any homogeneous function $f \in V$. Note that $\tilde{W}$ is a homogeneous operator with degree 2 in (2.24). 
It is not difficult to show that if $\tilde{D}$ is a degree operator, the partition function (2.24) can be generated by the $W$-representation. Unfortunately, it is clear that $\tilde{D}$ in $(2.24)$ is not the degree operator. It causes the usual $W$-representation of the matrix model (2.24) to fail here. In other words, the partition function (2.1) can not be obtained by acting on elementary functions with exponents of the operator $\tilde{W}$. In spite of this negative result, by evaluating the action of the homogeneous operator $\left(-\tilde{D}^{-1} \tilde{W}\right)^{k}$ on the function $e^{-\frac{\sqrt{\beta}}{\hbar} N t_{0}}$, we can still derive the compact expressions of the correlators from the representation (2.24). In what follows let us continue to discuss the correlators.

Since $\tilde{D}^{-1} \tilde{W}$ is an operator with degree 2 , we can see from $(2.24)$ that $Z_{G}^{(s)}=0$, when $s$ is odd. It leads to $\tilde{C}_{k_{1}, \cdots, k_{n}}^{s_{1}+\frac{1}{2}, \cdots, s_{m}+\frac{1}{2}}=0$, when $\sum_{\mu=1}^{n} k_{\mu}+\sum_{\nu=1}^{m} s_{\nu}+\frac{m}{2}=s$ is odd. In order to give the general expressions of the correlators for the $s$ even case, we need to write out the action $\left(-\tilde{D}^{-1} \tilde{W}\right)^{k} e^{-\frac{\sqrt{\beta}}{\hbar} N t_{0}}$ explicitly.

The operator $\tilde{D}^{-1}$ can be expressed as

$$
\begin{aligned}
\tilde{D}^{-1} & =\left(D-\frac{1}{2} \xi_{\frac{1}{2}} \frac{\partial}{\partial \xi_{\frac{1}{2}}}\right)^{-1} \\
& =D^{-1}+D^{-1}(2 D-1)^{-1} \xi_{\frac{1}{2}} \frac{\partial}{\partial \xi_{\frac{1}{2}}}
\end{aligned}
$$

where $D$ is the degree operator

$$
D=\sum_{k=1}^{\infty} k t_{k} \frac{\partial}{\partial t_{k}}+\sum_{k=0}^{\infty}\left(k+\frac{1}{2}\right) \xi_{k+\frac{1}{2}} \frac{\partial}{\partial \xi_{k+\frac{1}{2}}}
$$

and the relations $\left[D, \xi_{\frac{1}{2}} \frac{\partial}{\partial \xi_{\frac{1}{2}}}\right]=0$ and $\left(\xi_{\frac{1}{2}} \frac{\partial}{\partial \xi_{\frac{1}{2}}}\right)^{2}=\xi_{\frac{1}{2}} \frac{\partial}{\partial \xi_{\frac{1}{2}}}$ are used to give (2.25).

Thus the operators in (2.24) now take the following form:

$$
\begin{aligned}
\left(\tilde{D}^{-1} \tilde{W}\right)^{k} & =\left(D^{-1} \tilde{W}+D^{-1}(2 D-1)^{-1} \tilde{\mathbb{W}}\right)^{k} \\
& =\sum_{r=1}^{\left\lfloor\frac{k+1}{2}\right\rfloor} \sum_{\substack{l_{1}+\cdots+l_{2 r-1}=k \\
l_{1}, \cdots, l_{2 r-1} \geq 1}}\left(T_{1}+T_{2}\right)+\sum_{r=1}^{\left\lfloor\frac{k}{2}\right\rfloor} \sum_{\begin{array}{l}
l_{1}+\cdots+l_{2 r}=k \\
l_{1}, \cdots, l_{2 r} \geq 1
\end{array}}\left(T_{3}+T_{4}\right),
\end{aligned}
$$
where $\lfloor k\rfloor=\operatorname{Max}\{m \in \mathbb{Z} \mid m \leq k\}$ is the floor function, $\tilde{\mathbb{W}}=\xi_{\frac{1}{2}} \frac{\partial}{\partial \xi_{\frac{1}{2}}} \tilde{W}$, and $T_{i}, i=1, \cdots, 4$
are

$$
\begin{aligned}
& T_{1}=\left(D^{-1} \tilde{W}\right)^{l_{2 r-1}}\left(D^{-1}(2 D-1)^{-1} \tilde{\mathbb{W}}\right)^{l_{2 r-2}} \cdots\left(D^{-1}(2 D-1)^{-1} \tilde{\mathbb{W}}\right)^{l_{2}}\left(D^{-1} \tilde{W}\right)^{l_{1}}, \\
& T_{2}=\left(D^{-1}(2 D-1)^{-1} \tilde{\mathbb{W}}\right)^{l_{2 r-1}}\left(D^{-1} \tilde{W}\right)^{l_{2 r-2}} \cdots\left(D^{-1} \tilde{W}\right)^{l_{2}}\left(D^{-1}(2 D-1)^{-1} \tilde{\mathbb{W}}\right)^{l_{1}}, \\
& T_{3}=\left(D^{-1}(2 D-1)^{-1} \tilde{\mathbb{W}}\right)^{l_{2 r}}\left(D^{-1} \tilde{W}\right)^{l_{2 r-1}} \cdots\left(D^{-1}(2 D-1)^{-1} \tilde{\mathbb{W}}\right)^{l_{2}}\left(D^{-1} \tilde{W}\right)^{l_{1}}, \\
& T_{4}=\left(D^{-1} \tilde{W}\right)^{l_{2 r}}\left(D^{-1}(2 D-1)^{-1} \tilde{\mathbb{W}}\right)^{l_{2 r-1}} \cdots\left(D^{-1} \tilde{W}\right)^{l_{2}}\left(D^{-1}(2 D-1)^{-1} \tilde{\mathbb{W}}\right)^{l_{1}} .
\end{aligned}
$$


Using the fact that for the homogeneous function $f \in V, D^{-1} f=\operatorname{deg}(f)^{-1} f$, the action of the operator $T_{1}$ on the function $e^{-\frac{\sqrt{\beta}}{\hbar} N t_{0}}$ gives

$$
\begin{aligned}
& T_{1} e^{-\frac{\sqrt{\beta}}{\hbar} N t_{0}}=\frac{1}{k ! 2^{k}} \frac{1}{\prod_{i=l_{1}+1}^{k}(4 i-1)} \prod_{i=1}^{r-1} \prod_{j=1}^{l_{2 i+1}}\left[4\left(l_{1}+\cdots+l_{2 i}+j\right)-1\right] \\
& \text { - } \tilde{W}^{l_{2 r-1}} \tilde{\mathbb{W}}^{l_{2 r-2}} \ldots \tilde{\mathbb{W}}^{l_{2}} \tilde{W}^{l_{1}} e^{-\frac{\sqrt{\beta}}{\hbar} N t_{0}} \\
& =\frac{1}{k ! 2^{k}} \frac{1}{\prod_{i=l_{1}+1}^{k}(4 i-1)} \prod_{i=1}^{r-1} \prod_{j=1}^{l_{2 i+1}}\left[4\left(l_{1}+\cdots+l_{2 i}+j\right)-1\right] e^{-\frac{\sqrt{\beta}}{\hbar} N t_{0}}
\end{aligned}
$$

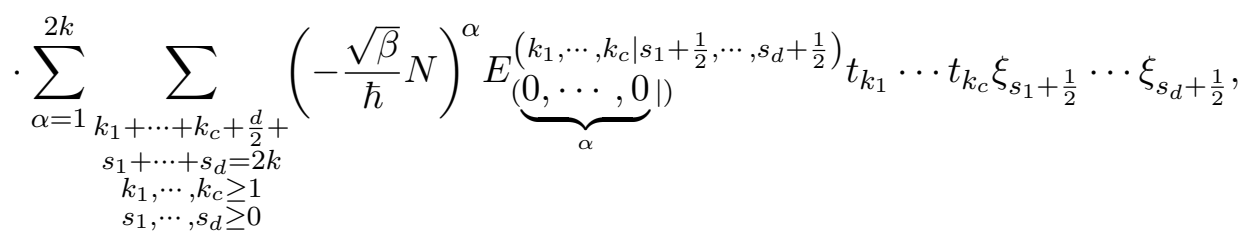

where we have used the formal expression of $\tilde{W}^{l_{2 r-1}} \tilde{\mathbb{W}}^{l_{2 r-2}} \ldots \tilde{\mathbb{W}}^{l_{2}} \tilde{W}^{l_{1}}$ with degree $2 k$

$$
\begin{aligned}
& \tilde{W}^{l_{2 r-1}} \tilde{\mathbb{W}}^{l_{2 r-2}} \cdots \tilde{\mathbb{W}}^{l_{2}} \tilde{W}^{l_{1}}=\sum_{a, c=0}^{2 k} \sum_{\begin{array}{c}
b, d=0 \\
i_{1}, \cdots, i_{a}=0 \\
j_{1}, \cdots, j_{b}=0
\end{array}}^{2 k+1} \sum_{\begin{array}{c}
k_{1}+\cdots+k_{c}+ \\
s_{1}+\cdots, s_{d}=\rho \\
k_{1}, \cdots, k_{c} \geq 1 \\
s_{1}, \cdots, s_{d} \geq 0
\end{array}}^{\infty} E_{\left(i_{1}, \cdots, i_{a} \mid j_{1}+\frac{1}{2}, \cdots, j_{b}+\frac{1}{2}\right)}^{\left(k_{1}, \cdots, k_{c} \mid s_{1}+\frac{1}{2}, \cdots, s_{d}+\frac{1}{2}\right)} t_{k_{1}} \cdots t_{k_{c}} \\
& \cdot \xi_{s_{1}+\frac{1}{2}} \cdots \xi_{s_{d}+\frac{1}{2}} \frac{\partial}{\partial t_{i_{1}}} \cdots \frac{\partial}{\partial t_{i_{a}}} \frac{\partial}{\partial \xi_{j_{1}+\frac{1}{2}}} \cdots \frac{\partial}{\partial \xi_{j_{b}+\frac{1}{2}}},
\end{aligned}
$$

$\rho=\sum_{\mu=1}^{a} i_{\mu}+\sum_{\nu=1}^{b} j_{\nu}+2 k+\frac{b}{2}-\frac{d}{2}$, the coefficients $E_{\left(i_{1}, \cdots, i_{a} \mid j_{1}+\frac{1}{2}, \cdots, j_{b}+\frac{1}{2}\right)}^{\left(k_{1}, \cdots, k_{c} \mid s_{1}+\frac{1}{2}, \cdots, s_{s}+\frac{1}{2}\right)}$ are polynomials with respect to $i_{\mu}, j_{\nu}, k_{\bar{\mu}}$ and $s_{\bar{\nu}}, \bar{\mu}=1, \cdots, c, \bar{\nu}=1, \cdots d$.

Similarly, we have

$$
\begin{aligned}
& T_{2} e^{-\frac{\sqrt{\beta}}{\hbar} N t_{0}}=\frac{1}{k ! 2^{k}} \frac{1}{\prod_{i=1}^{k}(4 i-1)} \prod_{i=1}^{r-1} \prod_{j=1}^{l_{2 i}}\left[4\left(l_{1}+\cdots+l_{2 i-1}+j\right)-1\right] e^{-\frac{\sqrt{\beta}}{\hbar} N t_{0}} \\
& \cdot \sum_{\substack{\alpha=1 \\
\begin{array}{c}
k_{1}+\cdots+k_{c}+\frac{d}{2}+\\
s_{1}+\cdots+s_{d}=2 k \\
k_{1}, \cdots, k_{c} \geq 1 \\
s_{1}, \cdots, s_{d} \geq 0
\end{array}}}^{2 k}\left(-\frac{\sqrt{\beta}}{\hbar} N\right)^{\alpha} F_{(\underbrace{0, \cdots, 0,0}_{\alpha} \mid)}^{\left(k_{1}, \cdots, k_{c} \mid s_{1}+\frac{1}{2}, \cdots, s_{d}+\frac{1}{2}\right)} t_{k_{1}} \cdots t_{k_{c}} \xi_{s_{1}+\frac{1}{2}} \cdots \xi_{s_{d}+\frac{1}{2}}, \\
& T_{3} e^{-\frac{\sqrt{\beta}}{\hbar} N t_{0}}=\frac{1}{k ! 2^{k}} \frac{1}{\prod_{i=l_{1}+1}^{k}(4 i-1)} \prod_{i=1}^{r-1} \prod_{j=1}^{l_{2 i+1}}\left[4\left(l_{1}+\cdots+l_{2 i}+j\right)-1\right] e^{-\frac{\sqrt{\beta}}{\hbar} N t_{0}} \\
& \cdot \sum_{\alpha=1}^{2 k} \sum_{\substack{k_{1}+\cdots+k_{c}+\frac{d}{2}+\\
s_{1}+\cdots+s_{d}=2 k \\
k_{1}, \cdots, k_{c} \geq 1 \\
s_{1}, \cdots, s_{d} \geq 0}}\left(-\frac{\sqrt{\beta}}{\hbar} N\right)^{\alpha} G_{(\underbrace{0, \cdots, 0}_{\alpha} \mid)}^{\left(k_{1}, \cdots, k_{c} \mid s_{1}+\frac{1}{2}, \cdots, s_{d}+\frac{1}{2}\right)} t_{k_{1}} \cdots t_{k_{c}} \xi_{s_{1}+\frac{1}{2}} \cdots \xi_{s_{d}+\frac{1}{2}} \text {, }
\end{aligned}
$$




$$
\begin{aligned}
& T_{4} e^{-\frac{\sqrt{\beta}}{\hbar} N t_{0}}=\frac{1}{k ! 2^{k}} \frac{1}{\prod_{i=1}^{k}(4 i-1)} \prod_{i=1}^{r} \prod_{j=1}^{l_{2 i}}\left[4\left(l_{1}+\cdots+l_{2 i-1}+j\right)-1\right] e^{-\frac{\sqrt{\beta}}{\hbar} N t_{0}} \\
& \cdot \sum_{\alpha=1}^{2 k} \sum_{\substack{k_{1}+\cdots+k_{c}+\frac{d}{2}+\\
s_{1}+\cdots+s_{d}=2 k \\
k_{1}, \cdots, k_{c} \geq 1 \\
s_{1}, \cdots, s_{d} \geq 0}}\left(-\frac{\sqrt{\beta}}{\hbar} N\right)^{\alpha} H_{\underbrace{\left(k_{1}, \cdots, k_{c} \mid s_{1}+\frac{1}{2}, \cdots, s_{d}+\frac{1}{2}\right)}_{\alpha}}^{(\underbrace{}_{k_{1}} \cdots t_{k_{c}} \xi_{s_{1}+\frac{1}{2}} \cdots \xi_{s_{d}+\frac{1}{2}}},
\end{aligned}
$$

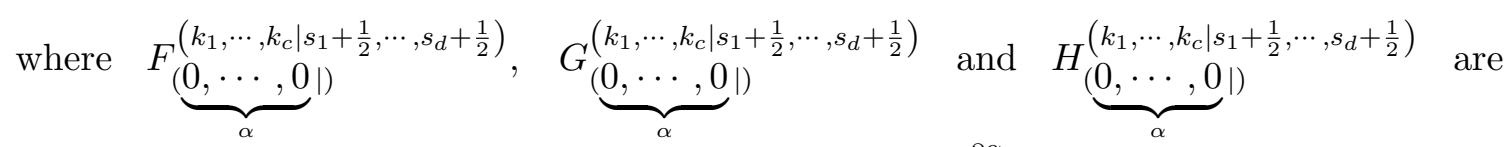
the coefficients of the terms $t_{k_{1}} \cdots t_{k_{c}} \xi_{s_{1}+\frac{1}{2}} \cdots \xi_{s_{d}+\frac{1}{2}} \frac{\partial^{\alpha}}{\partial t_{0}^{\alpha}}$ in the formal expansions of the corresponding operators $\tilde{\mathbb{W}}^{l_{2 r-1}} \tilde{W}^{l_{2 r-2}} \ldots \tilde{W}^{l_{2}} \tilde{\mathbb{W}}^{l_{1}}, \quad \tilde{\mathbb{W}}^{l_{2 r}} \tilde{W}^{l_{2 r-1}} \ldots \tilde{\mathbb{W}}^{l_{2}} \tilde{W}^{l_{1}}$ and $\tilde{W}^{l_{2 r}} \tilde{\mathbb{W}}^{l_{2 r-1}} \ldots \tilde{W}^{l_{2}} \tilde{\mathbb{W}}^{l_{1}}$, respectively.

Using the actions (2.29) and (2.31), we may give the coefficients of $t_{k_{1}} \cdots t_{k_{n}} \xi_{s_{1}+\frac{1}{2}} \cdots \xi_{s_{m}+\frac{1}{2}}$ with $\sum_{\mu=1}^{n} k_{\mu}+\sum_{\nu=1}^{m} s_{\nu}+\frac{m}{2}=2 k, k_{\mu} \geq 1, s_{\nu} \geq 0$ in (2.24). On the other hand, it is easy to give the corresponding coefficients in (2.13). Then from the equivalence of these coefficients, we obtain the desired expressions for the correlators

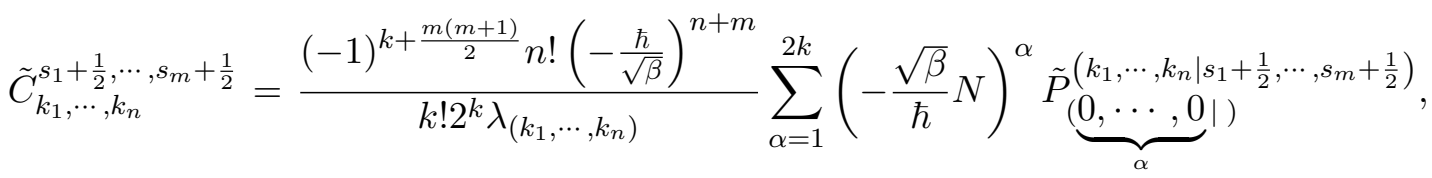

where $k=\frac{1}{2}\left(\sum_{\mu=1}^{n} k_{\mu}+\sum_{\nu=1}^{m} s_{\nu}+\frac{m}{2}\right), k_{\mu} \geq 1, s_{\nu} \geq 0, \lambda_{\left(k_{1}, \cdots, k_{n}\right)}$ is the number of distinct permutations of $\left(k_{1}, \cdots, k_{n}\right)$,

$$
\begin{aligned}
& \tilde{P}_{\left(k_{1}, \cdots, k_{n} \mid s_{1}+\frac{1}{2}, \cdots, s_{m}+\frac{1}{2}\right)}^{\left(k_{1}\right)} \\
& (\underbrace{0, \cdots, 0}_{\alpha} \mid) \\
& =\sum_{\sigma_{1}, \sigma_{2}}(-1)^{\tau\left(\sigma_{2}\left(s_{1}+\frac{1}{2}\right), \cdots, \sigma_{2}\left(s_{m}+\frac{1}{2}\right)\right)}\left[\sum _ { r = 1 } ^ { \lfloor \frac { k + 1 } { 2 } \rfloor } \sum _ { \begin{array} { c } 
{ l _ { 1 } + \cdots + l _ { 2 r - 1 } = k } \\
{ l _ { 1 } , \cdots , l _ { 2 r - 1 } \geq 1 }
\end{array} } \left(\frac{1}{\prod_{i=l_{1}+1}^{k}(4 i-1)}\right.\right. \\
& \cdot \prod_{i=1}^{r-1} \prod_{j=1}^{l_{2 i+1}}\left[4\left(l_{1}+\cdots+l_{2 i}+j\right)-1\right] E_{(\underbrace{0, \cdots, 0}_{\alpha} \mid)}^{\left(\sigma_{1}\left(k_{1}\right), \cdots, \sigma_{1}\left(k_{n}\right) \mid \sigma_{2}\left(s_{1}+\frac{1}{2}\right), \cdots, \sigma_{2}\left(s_{m}+\frac{1}{2}\right)\right)} \\
& \left.+\frac{1}{\prod_{i=1}^{k}(4 i-1)} \prod_{i=1}^{r-1} \prod_{j=1}^{l_{2 i}}\left[4\left(l_{1}+\cdots+l_{2 i-1}+j\right)-1\right] F_{(\underbrace{\left(\sigma_{1}\left(k_{1}\right), \cdots, \sigma_{1}\left(k_{n}\right) \mid \sigma_{2}\left(s_{1}+\frac{1}{2}\right), \cdots, \sigma_{2}\left(s_{m}+\frac{1}{2}\right)\right)}_{\alpha})}^{0,0}\right)
\end{aligned}
$$




$$
\begin{aligned}
& +\sum_{r=1}^{\left\lfloor\frac{k}{2}\right\rfloor} \sum_{\substack{l_{1}+\cdots+l_{2 r}=k \\
l_{1}, \cdots, l_{2 r} \geq 1}}\left(\frac{1}{\prod_{i=l_{1}+1}^{k}(4 i-1)} \prod_{i=1}^{r-1} \prod_{j=1}^{l_{2 i+1}}\left[4\left(l_{1}+\cdots+l_{2 i}+j\right)-1\right]\right. \\
& \cdot G_{(\underbrace{0, \cdots, 0}_{\alpha} \mid)}^{\left(\sigma_{1}\left(k_{1}\right), \cdots, \sigma_{1}\left(k_{n}\right) \mid \sigma_{2}\left(s_{1}+\frac{1}{2}\right), \cdots, \sigma_{2}\left(s_{m}+\frac{1}{2}\right)\right)}+\frac{1}{\prod_{i=1}^{k}(4 i-1)} \prod_{i=1}^{r} \prod_{j=1}^{l_{2 i}}\left[4\left(l_{1}+\cdots+l_{2 i-1}+j\right)-1\right] \\
& \left.\left.\cdot H_{(\underbrace{0, \cdots, 0}_{\alpha} \mid)}^{\left(\sigma_{1}\left(k_{1}\right), \cdots, \sigma_{1}\left(k_{n}\right) \mid \sigma_{2}\left(s_{1}+\frac{1}{2}\right), \cdots, \sigma_{2}\left(s_{m}+\frac{1}{2}\right)\right)}\right)\right] .
\end{aligned}
$$

Here $\sigma_{1}$ denotes all the distinct permutations of $\left(k_{1}, \cdots, k_{n}\right), \sigma_{2}$ is all the distinct permutations of $\left(s_{1}+\frac{1}{2}, \cdots, s_{m}+\frac{1}{2}\right)$ and its inverse number is denoted as $\tau\left(\sigma_{2}\left(s_{1}+\frac{1}{2}\right), \cdots, \sigma_{2}\left(s_{m}+\frac{1}{2}\right)\right)$.

Let us list the correlators for the case of $k=2$ in (2.32) as follows:

$$
\begin{aligned}
\tilde{C}_{4} & =\frac{\hbar^{2}}{4 \beta} N\left[2 \tilde{N}^{2}+(1-\beta) \tilde{N}+4 \beta\right], & \tilde{C}_{3,1} & =\frac{3 \hbar^{2}}{2 \beta} N \tilde{N}, \\
\tilde{C}_{2,2} & =\frac{\hbar^{2}}{4 \beta} N \tilde{N}(N \tilde{N}+4), & \tilde{C}_{1,1,1,1} & =\frac{3 \hbar^{2}}{\beta} N^{2}, \\
\tilde{C}_{2,1,1} & =\frac{\hbar^{2}}{2 \beta} N(N \tilde{N}+4), & C_{1,1}^{\frac{1}{2}, \frac{3}{2}} & =\frac{\hbar^{2}}{\beta} N^{2}, \\
\tilde{C}_{2}^{\frac{1}{2}, \frac{3}{2}} & =\frac{\hbar^{2}}{2 \beta} N(N \tilde{N}+4), & C_{1}^{\frac{1}{2}, \frac{5}{2}} & =\frac{2 \hbar^{2}}{\beta} N, \\
\tilde{C}^{\frac{1}{2}}, \frac{7}{2} & =\frac{\hbar^{2}}{2 \beta} N[3 \tilde{N}+2(1-\beta)], & \tilde{C}^{\frac{3}{2}, \frac{5}{2}} & =-\frac{\hbar^{2}}{2 \beta} N \tilde{N} .
\end{aligned}
$$

\section{Non-Gaussian supereigenvalue model in the Neveu-Schwarz sector}

Let us consider the non-Gaussian supereigenvalue model in the Neveu-Schwarz sector

$$
Z_{N G}(t, \xi ; a, \varepsilon)=\left(\prod_{i=1}^{N} \int_{-\infty}^{+\infty} d z_{i} \int d \theta_{i}\right) \Delta_{N S}(z, \theta)^{\beta} e^{-\frac{\sqrt{\beta}}{\hbar} \sum_{i=1}^{N}\left(V_{N S}\left(z_{i}, \theta_{i}\right)+\tilde{V}_{N S}\left(z_{i}, \theta_{i}\right)\right)},
$$

where $N$ is even, $\tilde{V}_{N S}(z, \theta)=\frac{1}{2 p+2} z^{2 p+2}+\sum_{k=1}^{2 p} \frac{1}{k} a_{k} z^{k}+\sum_{l=0}^{2 p} \varepsilon_{l} z^{l} \theta, p \geq 1, a_{k}$ and $\varepsilon_{l}$ are nonzero bosonic and fermionic coupling constants, respectively.

By applying the changes of integration variables $\left(z_{i} \rightarrow z_{i}+\epsilon \sum_{n=2 p+1}^{\infty} n t_{n} z_{i}^{n-2 p-1}, \theta_{i} \rightarrow\right.$ $\left.\theta_{i}+\frac{1}{2} \epsilon \sum_{n=2 p+1}^{\infty} n(n-2 p-1) t_{n} z_{i}^{n-2 p-2} \theta_{i}\right)$ and $\left(z_{i} \rightarrow z_{i}+\epsilon \sum_{n=2 p+1}^{\infty}\left(n+\frac{1}{2}\right) \xi_{n+\frac{1}{2}} z_{i}^{n-2 p-1} \theta_{i}\right.$, $\left.\theta_{i} \rightarrow \theta_{i}-\epsilon \sum_{n=2 p+1}^{\infty}\left(n+\frac{1}{2}\right) \xi_{n+\frac{1}{2}} z_{i}^{n-2 p-1}\right)$ for the partition function (3.1), we may derive the constraint from the invariance of the integral

$$
(\hat{D}+\hat{W}) Z_{N G}(t, \xi ; a, \varepsilon)=0
$$

where

$$
\hat{D}=\sum_{n=2 p+1}^{\infty} n t_{n} \frac{\partial}{\partial t_{n}}+\sum_{n=2 p+1}^{\infty}\left(n+\frac{1}{2}\right) \xi_{n+\frac{1}{2}} \frac{\partial}{\partial \xi_{n+\frac{1}{2}}}
$$


$\hat{W}=\hat{W}_{2 p+2}+\sum_{k=2}^{2 p+1} \hat{W}_{k}+\sum_{l=1}^{2 p+1} \hat{W}_{l+\frac{1}{2}}$, the operators $\hat{W}_{2 p+2}, \hat{W}_{k}$ and $\hat{W}_{l+\frac{1}{2}}$ are, respectively, given by

$$
\begin{aligned}
\hat{W}_{2 p+2}= & \sum_{n=2 p+1}^{\infty} n t_{n}\left[\sum_{k=1}^{\infty} k t_{k} \frac{\partial}{\partial t_{n+k-2 p-2}}+\frac{\hbar^{2}}{2} \sum_{k=0}^{n-2 p-2} \frac{\partial}{\partial t_{k}} \frac{\partial}{\partial t_{n-k-2 p-2}}\right. \\
& +\sum_{k=0}^{\infty}\left(\frac{n-1}{2}+k-p\right) \xi_{k+\frac{1}{2}} \frac{\partial}{\partial \xi_{k+n-2 p-\frac{3}{2}}}+\frac{\hbar^{2}}{2} \sum_{k=1}^{n-2 p-2} k \frac{\partial}{\partial \xi_{n-k-2 p-\frac{3}{2}}} \frac{\partial}{\partial \xi_{k-\frac{1}{2}}} \\
& \left.-\frac{\hbar}{2 \sqrt{\beta}}(1-\beta)(n-2 p-1) \frac{\partial}{\partial t_{n-2 p-2}}\right]+\sum_{n=2 p+1}^{\infty}\left(n+\frac{1}{2}\right) \xi_{n+\frac{1}{2}}\left[\sum_{k=1}^{\infty} k t_{k} \frac{\partial}{\partial \xi_{n+k-2 p-\frac{3}{2}}}\right. \\
& +\hbar^{2} \sum_{k=0}^{n-2 p-2} \frac{\partial}{\partial \xi_{k+\frac{1}{2}}} \frac{\partial}{\partial t_{n-k-2 p-2}}-\frac{\hbar}{\sqrt{\beta}}(1-\beta)(n-2 p-1) \frac{\partial}{\partial \xi_{n-2 p-\frac{3}{2}}} \\
& \left.+\sum_{k=0}^{\infty} \xi_{k+\frac{1}{2}} \frac{\partial}{\partial t_{k+n-2 p-1}}\right], \\
\hat{W}_{k}= & a_{2 p+2-k} \sum_{n=2 p+1}^{\infty} n t_{n} \frac{\partial}{\partial t_{n-k}}+a_{2 p+2-k} \sum_{n=2 p+1}^{\infty}\left(n+\frac{1}{2}\right) \xi_{n+\frac{1}{2}} \frac{\partial}{\partial \xi_{n+\frac{1}{2}-k}} \\
\hat{W}_{l+\frac{1}{2}}= & \varepsilon_{2 p+1-l} \sum_{n=2 p+1}^{\infty} n\left(p-l+\frac{n+1}{2}\right) t_{n} \frac{\partial}{\partial \xi_{n-\frac{1}{2}-l}} \\
& +\varepsilon_{2 p+1-l} \sum_{n=2 p+1}^{\infty}\left(n+\frac{1}{2}\right) \xi_{n+\frac{1}{2}} \frac{\partial}{\partial t_{n-l}} .
\end{aligned}
$$

The partition function (3.1) is now viewed as a vector in the space $\tilde{V}$ generated by the basis

$$
\left\{t_{k_{1}} \cdots t_{k_{n}} \xi_{s_{1}+\frac{1}{2}} \cdots \xi_{s_{m}+\frac{1}{2}} \mid k_{1}, \cdots, k_{n} \geq 1, s_{1}, \cdots, s_{m} \geq 0, n, m \in \mathbb{N}\right\}
$$

with coefficients on $\mathbb{C}\left[\left[t_{0}, a, \varepsilon\right]\right]$. Let us define the degrees of the coupling constants $a_{k}$ and $\varepsilon_{l}$ are 0 . Since $m \in \mathbb{N}$ in the basis (3.5), the partition function (3.1) is graded from $0, \frac{1}{2}, 1, \frac{3}{2}, \cdots, \infty$. Thus we have the expansion

$$
\begin{aligned}
Z_{N G}(t, \xi ; a, \varepsilon)= & e^{-\frac{\sqrt{\beta}}{\hbar} N t_{0}} Z_{N G}(a, \varepsilon)+\sum_{s \in \frac{1}{2} \mathbb{N}_{+}} Z_{N G}^{(s)}(t, \xi ; a, \varepsilon) \\
= & e^{-\frac{\sqrt{\beta}}{\hbar} N t_{0}}\left[Z_{N G}(a, \varepsilon)-\frac{\sqrt{\beta}}{\hbar} t_{k_{1}} C_{k_{1}}(a, \varepsilon)-\frac{\sqrt{\beta}}{\hbar} \xi_{s_{1}+\frac{1}{2}} C^{s_{1}+\frac{1}{2}}(a, \varepsilon)\right. \\
& +\frac{1}{2 !}\left(\frac{\sqrt{\beta}}{\hbar}\right)^{2} t_{k_{1}} t_{k_{2}} C_{k_{1}, k_{2}}(a, \varepsilon)+\left(\frac{\sqrt{\beta}}{\hbar}\right)^{2} t_{k_{1}} \xi_{s_{1}+\frac{1}{2}} C_{k_{1}}^{s_{1}+\frac{1}{2}}(a, \varepsilon) \\
& \left.-\frac{1}{2 !}\left(\frac{\sqrt{\beta}}{\hbar}\right)^{2} \xi_{s_{1}+\frac{1}{2}} \xi_{s_{2}+\frac{1}{2}} C^{s_{1}+\frac{1}{2}, s_{2}+\frac{1}{2}}(a, \varepsilon)+\cdots\right]
\end{aligned}
$$


where

$$
\begin{aligned}
Z_{N G}(a, \varepsilon)= & \left(\prod_{i=1}^{N} \int_{-\infty}^{+\infty} d z_{i} \int d \theta_{i}\right) \Delta_{N S}(z, \theta)^{\beta} e^{-\frac{\sqrt{\beta}}{\hbar} \sum_{i=1}^{N} \tilde{V}_{N S}\left(z_{i}, \theta_{i}\right)} \\
Z_{N G}^{(s)}(t, \xi ; a, \varepsilon)= & e^{-\frac{\sqrt{\beta}}{\hbar} N t_{0}}\left[\sum_{n=0}^{\infty} \sum_{m=0}^{\infty} \sum_{\substack{m \\
k_{1}+\cdots+k_{n}+\\
s_{1}+\cdots+s_{m}+\frac{m}{2}=s \\
k_{1}, \cdots, k_{n} \geq 1 \\
s_{1}, \cdots, s_{m} \geq 0}} \frac{(-1)^{\frac{m(m-1)}{2}}\left(-\frac{\sqrt{\beta}}{\hbar}\right)^{n+m}}{n ! m !} t_{k_{1}} \cdots t_{k_{n}}\right. \\
& \left.\cdot \xi_{s_{1}+\frac{1}{2}} \cdots \xi_{s_{m}+\frac{1}{2}} C_{k_{1}, \cdots, k_{n}}^{s_{1}+\frac{1}{2}, \cdots, s_{m}+\frac{1}{2}}(a, \varepsilon)\right]
\end{aligned}
$$

and the correlators $C_{k_{1}, \cdots, k_{n}}^{s_{1}+\frac{1}{2}, \cdots, s_{m}+\frac{1}{2}}(a, \varepsilon)$ are defined by

$$
\begin{aligned}
C_{k_{1}, \cdots, k_{n}}^{s_{1}+\frac{1}{2}, \cdots, s_{m}+\frac{1}{2}}(a, \varepsilon)= & \left(\prod_{i=1}^{N} \int_{-\infty}^{+\infty} d z_{i} \int d \theta_{i}\right) \sum_{\substack{i_{1}, \cdots, i_{n}=1 \\
j_{1}, \cdots, j_{m}=1}}^{N} z_{i_{1}}^{k_{1}} \cdots z_{i_{n}}^{k_{n}} z_{j_{1}}^{s_{1}} \theta_{j_{1}} \cdots z_{j_{m}}^{s_{m}} \theta_{j_{m}} \\
& \cdot \Delta_{N S}(z, \theta)^{\beta} e^{-\frac{\sqrt{\beta}}{\hbar} \sum_{i=1}^{N} \tilde{V}_{N S}\left(z_{i}, \theta_{i}\right)} .
\end{aligned}
$$

It should be noted that the correlators $C_{k_{1}, \cdots, k_{n}}^{s_{1}+\frac{1}{2}, \cdots, s_{m}+\frac{1}{2}}(a, \varepsilon)$ with the fermionic coupling constants are written on the right side of $t_{k_{1}} \cdots t_{k_{n}} \xi_{s_{1}+\frac{1}{2}} \cdots \xi_{s_{m}+\frac{1}{2}}$ in (3.6) for the convenience of the following discussions.

Let us further analyze the constraint (3.2). We observe that in (3.2) $\hat{D}=D-$ $\sum_{k=1}^{2 p} k t_{k} \frac{\partial}{\partial t_{k}}-\sum_{l=0}^{2 p}\left(l+\frac{1}{2}\right) \xi_{l+\frac{1}{2}} \frac{\partial}{\partial \xi_{l+\frac{1}{2}}}$ has degree $0, \hat{W}_{2 p+2}, \hat{W}_{k}$ and $\hat{W}_{l+\frac{1}{2}}$ are operators with degrees $2 p+2, k$ and $l+\frac{1}{2}$, respectively. Since $\operatorname{Ker}(\hat{D}+\hat{W})$ is no longer one dimensional on $\tilde{V}$, the partition function (3.1) can not be uniquely determined by the constraint (3.2). On the other hand, there are the additional constraints for the partition function (3.1)

$$
\begin{aligned}
\frac{\partial}{\partial t_{k}} Z_{N G}(t, \xi ; a, \varepsilon) & =k \frac{\partial}{\partial a_{k}} Z_{N G}(t, \xi ; a, \varepsilon), & & k=1, \cdots, 2 p, \\
\frac{\partial}{\partial \xi_{l+\frac{1}{2}}} Z_{N G}(t, \xi ; a, \varepsilon) & =\frac{\partial}{\partial \varepsilon_{l}} Z_{N G}(t, \xi ; a, \varepsilon), & l & =0, \cdots, 2 p .
\end{aligned}
$$

Substituting (3.10) into (3.2), we obtain

$$
(D+\hat{\mathcal{W}}) Z_{N G}(t, \xi ; a, \varepsilon)=0,
$$

where $\hat{\mathcal{W}}=\hat{W}-\sum_{k=1}^{2 p} k^{2} t_{k} \frac{\partial}{\partial a_{k}}-\sum_{l=0}^{2 p}\left(l+\frac{1}{2}\right) \xi_{l+\frac{1}{2}} \frac{\partial}{\partial \varepsilon_{l}}$.

Since the operators $D$ and $D+\hat{\mathcal{W}}$ are invertible on $-\hat{\mathcal{W}} e^{-\frac{\sqrt{\beta}}{\hbar} N t_{0}} Z_{N G}(a, \varepsilon)$, by the constraint (3.11), we have

$$
\sum_{s \in \frac{1}{2} \mathbb{N}_{+}}^{\infty} Z_{N G}^{(s)}(t, \xi ; a, \varepsilon)=-(D+\hat{\mathcal{W}})^{-1} \hat{\mathcal{W}} e^{-\frac{\sqrt{\beta}}{\hbar} N t_{0}}
$$


Hence the partition function (3.1) can be expressed as

$$
Z_{N G}(t, \xi ; a, \varepsilon)=\sum_{k=0}^{\infty}\left(-D^{-1} \hat{\mathcal{W}}\right)^{k} e^{-\frac{\sqrt{\beta}}{\hbar} N t_{0}} Z_{N G}(a, \varepsilon)
$$

Similar to the representation for the non-Gaussian Hermitian matrix model presented in ref. [40], we see that $\hat{\mathcal{W}}$ in (3.13) is not a homogeneous operator. Since $\hat{\mathcal{W}}$ contains the noncommutative operators with degrees ranging from $\frac{1}{2}$ to $2 p+2$, it not only leads to the fact that the partition function (3.1) can not be obtained by acting on elementary functions with exponents of the operator $\hat{\mathcal{W}}$, but also makes the handling of the correlators quite difficult from (3.13). We can in principle derive the correlators step by step from (3.13).

For example, we give some correlators as follows:

$$
\begin{array}{rlrl}
C^{\frac{1}{2}}(a, \varepsilon) & =-\frac{\hbar}{\sqrt{\beta}} \frac{\partial}{\partial \varepsilon_{0}} Z_{N G}(a, \varepsilon), & C_{1}(a, \varepsilon) & =-\frac{\hbar}{\sqrt{\beta}} \frac{\partial}{\partial a_{1}} Z_{N G}(a, \varepsilon), \\
C_{1}^{\frac{1}{2}}(a, \varepsilon) & =\frac{\hbar^{2}}{\beta} \frac{\partial^{2}}{\partial a_{1} \partial \varepsilon_{0}} Z_{N G}(a, \varepsilon), & C^{\frac{3}{2}}(a, \varepsilon) & =-\frac{\hbar}{\sqrt{\beta}} \frac{\partial}{\partial \varepsilon_{1}} Z_{N G}(a, \varepsilon), \\
C_{2}(a, \varepsilon) & =-\frac{2 \hbar}{\sqrt{\beta}} \frac{\partial}{\partial a_{2}} Z_{N G}(a, \varepsilon), & C_{1,1}(a, \varepsilon) & =\frac{\hbar^{2}}{\beta} \frac{\partial^{2}}{\partial a_{1}^{2}} Z_{N G}(a, \varepsilon), \\
C^{\frac{1}{2}, \frac{3}{2}}(a, \varepsilon) & =\frac{2 \hbar^{2}}{\beta} \frac{\partial^{2}}{\partial \varepsilon_{0} \partial \varepsilon_{1}} Z_{N G}(a, \varepsilon), & C_{2}^{\frac{1}{2}}(a, \varepsilon)=\frac{2 \hbar^{2}}{\beta} \frac{\partial^{2}}{\partial a_{2} \partial \varepsilon_{0}} Z_{N G}(a, \varepsilon), \\
C_{1,1}^{\frac{1}{2}}(a, \varepsilon) & =-\left(\frac{\hbar}{\sqrt{\beta}}\right)^{3} \frac{\partial^{3}}{\partial a_{1}^{2} \partial \varepsilon_{0}} Z_{N G}(a, \varepsilon), & C_{1}^{\frac{3}{2}}(a, \varepsilon)=\frac{\hbar^{2}}{\beta} \frac{\partial^{2}}{\partial a_{1} \partial \varepsilon_{1}} Z_{N G}(a, \varepsilon), \\
C^{\frac{5}{2}}(a, \varepsilon) & =-\frac{\hbar}{\sqrt{\beta}} \frac{\partial}{\partial \varepsilon_{2}} Z_{N G}(a, \varepsilon), & C_{2,1}(a, \varepsilon)=\frac{4 \hbar^{2}}{\beta} \frac{\partial^{2}}{\partial a_{1} \partial a_{2}} Z_{N G}(a, \varepsilon), \\
C_{1,1,1}(a, \varepsilon) & =-\left(\frac{\hbar}{\sqrt{\beta}}\right)^{3} \frac{\partial^{3}}{\partial a_{1}^{3}} Z_{N G}(a, \varepsilon), & C^{\frac{1}{2}, \frac{5}{2}}(a, \varepsilon)=\frac{2 \hbar^{2}}{\beta} \frac{\partial^{2}}{\partial \varepsilon_{0} \partial \varepsilon_{2}} Z_{N G}(a, \varepsilon), \\
C_{3}(a, \varepsilon) & = \begin{cases}{\left[-N a_{1}+\frac{\hbar}{\sqrt{\beta}}\left(a_{2} \frac{\partial}{\partial a_{1}}+\varepsilon_{1} \frac{\partial}{\partial \varepsilon_{0}}+2 \varepsilon_{2} \frac{\partial}{\partial \varepsilon_{1}}\right)\right] Z_{N G}(a, \varepsilon), p=1 ;} \\
-\frac{3 \hbar}{\sqrt{\beta}} \frac{\partial}{\partial a_{3}} Z_{N G}(a, \varepsilon),\end{cases} \\
C_{1}^{\frac{1}{2}, \frac{3}{2}}(a, \varepsilon) & =-2\left(\frac{\hbar}{\sqrt{\beta}}\right)^{3} \frac{\partial^{3}}{\partial a_{1} \partial \varepsilon_{0} \partial \varepsilon_{1}} Z_{N G}(a, \varepsilon) .
\end{array}
$$

\section{Chiral supereigenvalue model in the Neveu-Schwarz sector}

Let us consider the chiral supereigenvalue model in the Neveu-Schwarz sector

$$
Z_{C}=\frac{1}{\Lambda_{C}}\left(\prod_{i=1}^{N} \int_{0}^{+\infty} d z_{i} \int d \theta_{i}\right) \Delta_{N S}(z, \theta)^{\beta} e^{-\frac{\sqrt{\beta}}{\hbar} \sum_{i=1}^{N}\left(V_{N S}\left(z_{i}, \theta_{i}\right)+z_{i}\right)}
$$

where $N$ is even, the normalization factor $\Lambda_{C}$ is given by

$$
\Lambda_{C}=\left(\prod_{i=1}^{N} \int_{0}^{+\infty} d z_{i} \int d \theta_{i}\right) \Delta_{N S}(z, \theta)^{\beta} e^{-\frac{\sqrt{\beta}}{\hbar} \sum_{i=1}^{N} z_{i}}
$$


The correlators $\bar{C}_{k_{1}, \cdots, k_{n}}^{s_{1}+\frac{1}{2}, \cdots, s_{m}+\frac{1}{2}}$ in the chiral supereigenvalue model are defined by

$$
\begin{aligned}
\bar{C}_{k_{1}, \cdots, k_{n}}^{s_{1}+\frac{1}{2}, \cdots, s_{m}+\frac{1}{2}}= & \frac{1}{\Lambda_{C}}\left(\prod_{i=1}^{N} \int_{0}^{+\infty} d z_{i} \int d \theta_{i}\right) \sum_{\substack{i_{1}, \cdots, i_{n}=1 \\
j_{1}, \cdots, j_{m}=1}}^{N} z_{i_{1}}^{k_{1}} \cdots z_{i_{n}}^{k_{n}} z_{j_{1}}^{s_{1}} \theta_{j_{1}} \cdots z_{j_{m}}^{s_{m}} \theta_{j_{m}} \\
& \cdot \Delta_{N S}(z, \theta)^{\beta} e^{-\frac{\sqrt{\beta}}{\hbar} \sum_{i=1}^{N} z_{i}} .
\end{aligned}
$$

Note that (4.3) is not convergent when the bosonic variables $z_{i}$ are integrated from $-\infty$ to $+\infty$. This is the reason why we consider the chiral case (4.1) instead of the supereigenvalue model (1.2).

By the invariance of the partition function (4.1) under two pairs of the changes of integration variables $\left(z_{i} \rightarrow z_{i}+\epsilon \sum_{n=1}^{\infty} n t_{n} z_{i}^{n}, \quad \theta_{i} \rightarrow \theta_{i}+\frac{1}{2} \epsilon \sum_{n=1}^{\infty} n^{2} t_{n} z_{i}^{n-1} \theta_{i}\right)$ and $\left(z_{i} \rightarrow\right.$ $\left.z_{i}+\epsilon \sum_{n=1}^{\infty}\left(n+\frac{1}{2}\right) \xi_{n+\frac{1}{2}} z_{i}^{n} \theta_{i}, \quad \theta_{i} \rightarrow \theta_{i}-\epsilon \sum_{n=1}^{\infty}\left(n+\frac{1}{2}\right) \xi_{n+\frac{1}{2}} z_{i}^{n}\right)$, we obtain

$$
(\tilde{D}+\bar{W}) Z_{C}=0
$$

where

$$
\begin{aligned}
\bar{W}= & \sum_{n, k=1}^{\infty} n k t_{n} t_{k} \frac{\partial}{\partial t_{n+k-1}}+\sum_{n, k=1}^{\infty} n\left(2 k+\frac{n+1}{2}\right) t_{n} \xi_{k+\frac{1}{2}} \frac{\partial}{\partial \xi_{n+k-\frac{1}{2}}} \\
& +\frac{1}{2} \sum_{n=1}^{\infty} n^{2} t_{n} \xi_{\frac{1}{2}} \frac{\partial}{\partial \xi_{n-\frac{1}{2}}}+\frac{\hbar^{2}}{2} \sum_{n=1}^{\infty} \sum_{k=0}^{n-1} n t_{n} \frac{\partial}{\partial t_{k}} \frac{\partial}{\partial t_{n-k-1}} \\
& +\frac{\hbar^{2}}{2} \sum_{n=2}^{\infty} \sum_{k=1}^{n-1} n k t_{n} \frac{\partial}{\partial \xi_{n-k-\frac{1}{2}}} \frac{\partial}{\partial \xi_{k-\frac{1}{2}}}-\frac{\hbar}{2 \sqrt{\beta}}(1-\beta) \sum_{n=1}^{\infty} n^{2} t_{n} \frac{\partial}{\partial t_{n-1}} \\
& +\hbar^{2} \sum_{n=1}^{\infty} \sum_{k=0}^{n-1}\left(n+\frac{1}{2}\right) \xi_{n+\frac{1}{2}} \frac{\partial}{\partial \xi_{k+\frac{1}{2}}} \frac{\partial}{\partial t_{n-k-1}}+\sum_{n=1}^{\infty} \sum_{k=0}^{\infty}\left(n+\frac{1}{2}\right) \xi_{n+\frac{1}{2}} \xi_{k+\frac{1}{2}} \frac{\partial}{\partial t_{n+k}} \\
& -\frac{\hbar}{\sqrt{\beta}}(1-\beta) \sum_{n=1}^{\infty} n\left(n+\frac{1}{2}\right) \xi_{n+\frac{1}{2}} \frac{\partial}{\partial \xi_{n-\frac{1}{2}}} .
\end{aligned}
$$

Some specific types of the correlators can be recursively derived from the constraint (4.4). Taking the coefficients of $t_{1}^{l+1}, t_{1}^{l} \xi_{\frac{1}{2}} \xi_{\frac{3}{2}}, t_{1}^{l} t_{2}$ in (4.4) and setting to zero, respectively, we obtain

$$
\bar{C}_{1}=\frac{\hbar}{2 \sqrt{\beta}} N \tilde{N},
$$

and the recursive relations

$$
\begin{aligned}
\bar{C}_{\underbrace{1, \cdots, 1}_{l+1}} & =\frac{\hbar}{2 \sqrt{\beta}}(N \tilde{N}+2 l) \bar{C}_{\underbrace{1, \cdots, 1}_{l}}, \\
\bar{C}_{\underbrace{\frac{1}{2}, \frac{3}{2}}_{l}, \cdots, 1}^{1, \cdots,} & =\frac{\hbar}{\sqrt{\beta}(2 l+3)}[l(2 l+5+N \tilde{N}) \underbrace{\bar{C}_{\underbrace{2}, \frac{3}{2}}^{1, \cdots, 1}}_{l}+3 \bar{C}_{\underbrace{1, \cdots, 1}_{l-1}}], \\
\bar{C}_{2, \underbrace{1, \cdots, 1}_{l+1}} & =\frac{\hbar}{\sqrt{\beta}(l+2)}\left[l\left(l+3+\frac{1}{2} N \tilde{N}\right) \bar{C}_{2, \underbrace{1, \cdots, 1}_{l-1}}^{\overline{1}_{l}, \cdots \tilde{N} \bar{C}_{1, \cdots, 1}^{1, \cdots, 1}}\right] .
\end{aligned}
$$


From (4.6) and (4.7), we can further derive the correlators

$$
\begin{aligned}
\bar{C}_{\underbrace{1, \cdots, 1}_{l}} & =\left(\frac{\hbar}{\sqrt{\beta}}\right)^{l} \prod_{j=0}^{l-1}\left(\frac{N \tilde{N}}{2}+j\right), \\
\bar{C}_{\underbrace{1, \frac{3}{2}, \cdots, 1}_{l}}^{\underbrace{\frac{3}{2} \cdots,}} & =\left(\frac{\hbar}{\sqrt{\beta}}\right)^{l+2} \frac{N \tilde{N}}{2} \prod_{j=1}^{l}\left(\frac{N \tilde{N}}{2}+j+1\right), \\
\bar{C}_{2, \underbrace{1, \cdots, 1}_{l}} & =\left(\frac{\hbar}{\sqrt{\beta}}\right)^{l+2} \frac{N \tilde{N}^{2}}{2} \prod_{j=1}^{l}\left(\frac{N \tilde{N}}{2}+j+1\right) .
\end{aligned}
$$

We observe that the operator $\tilde{D}$ in (4.4) is the same as in (2.12), and the operator $\bar{W}$ in (4.4) is a homogeneous operator with degree 1. Following the similar considerations in the Gaussian supereigenvalue model, we reach the final expressions for the partition function (4.1)

$$
Z_{C}=\sum_{k=0}^{\infty}\left(-\tilde{D}^{-1} \bar{W}\right)^{k} e^{-\frac{\sqrt{\beta}}{\hbar} N t_{0}}
$$

and the correlators

$$
\bar{C}_{k_{1}, \cdots, k_{n}}^{s_{1}+\frac{1}{2}, \cdots, s_{m}+\frac{1}{2}}=\frac{(-1)^{s+\frac{m(m+1)}{2}} n !\left(-\frac{\hbar}{\sqrt{\beta}}\right)^{n+m}}{s ! \lambda_{\left(k_{1}, \cdots, k_{n}\right)}} \sum_{\alpha=1}^{2 s}\left(-\frac{\sqrt{\beta}}{\hbar} N\right)^{\alpha} \bar{P}_{(\underbrace{\left(k_{1}, \cdots, k_{n} \mid s_{1}+\frac{1}{2}, \cdots, s_{m}+\frac{1}{2}\right.}_{\alpha})}^{(\underbrace{}_{\alpha} \mid)},
$$

where $s=\sum_{\mu=1}^{n} k_{\mu}+\sum_{\nu=1}^{m} s_{\nu}+\frac{m}{2}, k_{\mu} \geq 1, s_{\nu} \geq 0$,

$$
\begin{aligned}
& \bar{P}_{(\underbrace{\left(k_{1}, \cdots, k_{n} \mid s_{1}+\frac{1}{2}, \cdots, s_{m}+\frac{1}{2}\right)}_{\alpha})}^{(\underbrace{}_{\alpha}, 0)} \\
& =\sum_{\sigma_{1}, \sigma_{2}}(-1)^{\tau\left(\sigma_{2}\left(s_{1}+\frac{1}{2}\right), \cdots, \sigma_{2}\left(s_{m}+\frac{1}{2}\right)\right)}\left[\sum _ { r = 1 } ^ { \lfloor \frac { s + 1 } { 2 } \rfloor } \sum _ { \substack { l _ { 1 } + \cdots + l _ { 2 r - 1 } = s \\
l _ { 1 } , \cdots , l _ { 2 r - 1 } \geq 1 } } \left(\frac{1}{\prod_{i=l_{1}+1}^{s}(2 i-1)}\right.\right. \\
& \cdot \prod_{i=1}^{r-1} \prod_{j=1}^{l_{2 i+1}}\left[2\left(l_{1}+\cdots+l_{2 i}+j\right)-1\right] \bar{E}_{(\underbrace{0, \cdots, 0}_{\alpha} \mid)}^{\left(\sigma_{1}\left(k_{1}\right), \cdots, \sigma_{1}\left(k_{n}\right) \mid \sigma_{2}\left(s_{1}+\frac{1}{2}\right), \cdots, \sigma_{2}\left(s_{m}+\frac{1}{2}\right)\right)} \\
& \left.+\frac{1}{\prod_{i=1}^{s}(2 i-1)} \prod_{i=1}^{r-1} \prod_{j=1}^{l_{2 i}}\left[2\left(l_{1}+\cdots+l_{2 i-1}+j\right)-1\right] \bar{F}_{(\underbrace{0, \cdots, 0}_{\alpha} \mid)}^{\left(\sigma_{1}\left(k_{1}\right), \cdots, \sigma_{1}\left(k_{n}\right) \mid \sigma_{2}\left(s_{1}+\frac{1}{2}\right), \cdots, \sigma_{2}\left(s_{m}+\frac{1}{2}\right)\right)}\right) \\
& +\sum_{r=1}^{\left\lfloor\frac{s}{2}\right\rfloor} \sum_{\substack{l_{1}+\cdots+l_{2 r}=s \\
l_{1}, \cdots, l_{2 r} \geq 1}}\left(\frac{1}{\prod_{i=l_{1}+1}^{s}(2 i-1)} \prod_{i=1}^{r-1} \prod_{j=1}^{l_{2 i+1}}\left[2\left(l_{1}+\cdots+l_{2 i}+j\right)-1\right]\right.
\end{aligned}
$$




$$
\begin{aligned}
& \cdot \bar{G}_{(\underbrace{0, \cdots, 0}_{\alpha} \mid)}^{\left(\sigma_{1}\left(k_{1}\right), \cdots, \sigma_{1}\left(k_{n}\right) \mid \sigma_{2}\left(s_{1}+\frac{1}{2}\right), \cdots, \sigma_{2}\left(s_{m}+\frac{1}{2}\right)\right)}+\frac{1}{\prod_{i=1}^{s}(2 i-1)} \prod_{i=1}^{r} \prod_{j=1}^{l_{2 i}}\left[2\left(l_{1}+\cdots+l_{2 i-1}+j\right)-1\right] \\
& \cdot \bar{H}_{\left.(\underbrace{\left(\sigma_{1}\left(k_{1}\right), \cdots, \sigma_{1}\left(k_{n}\right) \mid \sigma_{2}\left(s_{1}+\frac{1}{2}\right), \cdots, \sigma_{2}\left(s_{m}+\frac{1}{2}\right)\right)}_{\alpha})\right]}^{0, \cdots, 0} \mid
\end{aligned}
$$

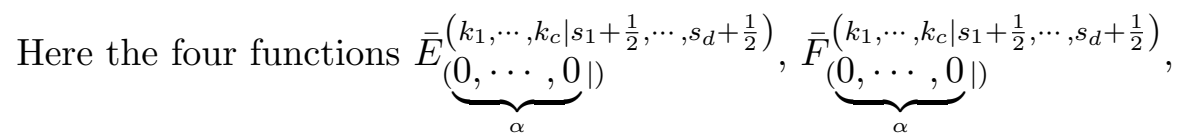

$\bar{G}_{(\underbrace{\left(k_{1}, \cdots, k_{c} \mid s_{1}+\frac{1}{2}, \cdots, s_{d}+\frac{1}{2}\right)}_{\alpha})}$ and $\left.\bar{H}_{(\underbrace{\left(k_{1}, \cdots, k_{c} \mid s_{1}+\frac{1}{2}, \cdots, s_{d}+\frac{1}{2}\right)}_{\alpha})}{ }^{\alpha} \mid\right)$ are, respectively, the coefficients of $t_{k_{1}} \cdots t_{k_{c}} \xi_{s_{1}+\frac{1}{2}} \cdots \xi_{s_{d}+\frac{1}{2}} \frac{\partial^{\alpha}}{\partial t_{0}^{\alpha}}$ in the formal expansions of the corresponding operators $\bar{W}^{l_{2 r-1}} \overline{\mathbb{W}}^{l_{2 r-2}} \ldots \overline{\mathbb{W}}^{l_{2}} \bar{W}^{l_{1}}, \overline{\mathbb{W}}^{l_{2 r-1}} \bar{W}^{l_{2 r-2}} \ldots \bar{W}^{l_{2}} \overline{\mathbb{W}}^{l_{1}}, \overline{\mathbb{W}}^{l_{2 r}} \bar{W}^{l_{2 r-1}} \ldots \overline{\mathbb{W}}^{l_{2}} \bar{W}^{l_{1}}$ and $\bar{W}^{l_{2 r}} \overline{\mathbb{W}}^{l_{2 r-1}}$ $\cdots \bar{W}^{l_{2}} \overline{\mathbb{W}}^{l_{1}}$, the operator $\overline{\mathbb{W}}$ is given by $\overline{\mathbb{W}}=\xi_{\frac{1}{2}} \frac{\partial}{\partial \xi_{\frac{1}{2}}} \bar{W}$.

Let us list the correlators (4.10) with $\sum_{\mu=1}^{n} k_{\mu}+\sum_{\nu=1}^{m} s_{\nu}+\frac{m}{2} \leq 3$ as follows:

$$
\begin{array}{rlrl}
\bar{C}_{1} & =\frac{\hbar}{2 \sqrt{\beta}} N \tilde{N}, & \bar{C}_{2}=\frac{\hbar^{2}}{2 \beta} N \tilde{N}^{2}, \\
\bar{C}_{1,1} & =\frac{\hbar^{2}}{4 \beta} N \tilde{N}(N \tilde{N}+2), \quad \bar{C}^{\frac{1}{2}, \frac{3}{2}}=\frac{\hbar^{2}}{2 \beta} N \tilde{N}, \\
\bar{C}_{3} & =\frac{1}{8}\left(\frac{\hbar}{\sqrt{\beta}}\right)^{3} N \tilde{N}\left[4 \beta+\tilde{N}(1-\beta)+5 \tilde{N}^{2}\right], \\
\bar{C}_{2,1} & =\frac{1}{4}\left(\frac{\hbar}{\sqrt{\beta}}\right)^{3} N \tilde{N}^{2}(N \tilde{N}+4), \\
\bar{C}_{1,1,1} & =\frac{1}{8}\left(\frac{\hbar}{\sqrt{\beta}}\right)^{3} N \tilde{N}(N \tilde{N}+2)(N \tilde{N}+4), \\
\bar{C}_{1}^{\frac{1}{2}, \frac{3}{2}} & =\frac{1}{4}\left(\frac{\hbar}{\sqrt{\beta}}\right)^{3} N \tilde{N}(N \tilde{N}+4), \\
\bar{C}^{\frac{1}{2}, \frac{5}{2}} & =\frac{1}{2}\left(\frac{\hbar}{\sqrt{\beta}}\right)^{3} N \tilde{N}(2 \tilde{N}+1-\beta) .
\end{array}
$$

\section{Non-Gaussian chiral supereigenvalue model in the Neveu-Schwarz sec- tor}

The non-Gaussian chiral supereigenvalue model in the Neveu-Schwarz sector is

$$
Z_{C N G}(t, \xi ; a, \varepsilon)=\left(\prod_{i=1}^{N} \int_{0}^{+\infty} d z_{i} \int d \theta_{i}\right) \Delta_{N S}(z, \theta)^{\beta} e^{-\frac{\sqrt{\beta}}{\hbar} \sum_{i=1}^{N}\left(V_{N S}\left(z_{i}, \theta_{i}\right)+\bar{V}_{N S}\left(z_{i}, \theta_{i}\right)\right)},
$$

where $N$ is even, $\bar{V}_{N S}(z, \theta)=\frac{1}{2 p+1} z^{2 p+1}+\sum_{k=1}^{2 p} \frac{1}{k} a_{k} z^{k}+\sum_{l=0}^{2 p} \varepsilon_{l} z^{l} \theta, p \geq 1, a_{k}$ and $\varepsilon_{l}$ are nonzero bosonic and fermionic coupling constants, respectively. The correlators 
$\hat{C}_{k_{1}, \cdots, k_{n}}^{s_{1}+\frac{1}{2}, \cdots, s_{m}+\frac{1}{2}}(a, \varepsilon)$ are defined by

$$
\begin{aligned}
\hat{C}_{k_{1}, \cdots, k_{n}}^{s_{1}+\frac{1}{2}, \cdots, s_{m}+\frac{1}{2}}(a, \varepsilon)= & \left(\prod_{i=1}^{N} \int_{0}^{+\infty} d z_{i} \int d \theta_{i}\right) \sum_{\substack{i_{1}, \cdots, i_{n}=1 \\
j_{1}, \cdots, j_{m}=1}}^{N} z_{i_{1}}^{k_{1}} \cdots z_{i_{n}}^{k_{n}} z_{j_{1}}^{s_{1}} \theta_{j_{1}} \cdots z_{j_{m}}^{s_{m}} \theta_{j_{m}} \\
& \cdot \Delta_{N S}(z, \theta)^{\beta} e^{-\frac{\sqrt{\beta}}{\hbar} \sum_{i=1}^{N} \bar{V}_{N S}\left(z_{i}, \theta_{i}\right)} .
\end{aligned}
$$

By applying the changes of integration variables $\left(z_{i} \rightarrow z_{i}+\epsilon \sum_{n=2 p+1}^{\infty} n t_{n} z_{i}^{n-2 p}\right.$, $\left.\theta_{i} \rightarrow \theta_{i}+\frac{1}{2} \epsilon \sum_{n=2 p+1}^{\infty} n(n-2 p) t_{n} z_{i}^{n-2 p-1} \theta_{i}\right)$ and $\left(z_{i} \rightarrow z_{i}+\epsilon \sum_{n=2 p+1}^{\infty}\left(n+\frac{1}{2}\right) \xi_{n+\frac{1}{2}} z_{i}^{n-2 p} \theta_{i}\right.$, $\left.\theta_{i} \rightarrow \theta_{i}-\epsilon \sum_{n=2 p+1}^{\infty}\left(n+\frac{1}{2}\right) \xi_{n+\frac{1}{2}} z_{i}^{n-2 p}\right)$ for the partition function (5.1), we may derive the constraint from the invariance of the integral

$$
(\hat{D}+\check{W}) Z_{C N G}(t, \xi ; a, \varepsilon)=0,
$$

where $\check{W}=\check{W}_{2 p+1}+\sum_{k=1}^{2 p} \check{W}_{k}+\sum_{l=0}^{2 p} \check{W}_{l+\frac{1}{2}}$, the operators $\check{W}_{2 p+1}, \check{W}_{k}$ and $\check{W}_{l+\frac{1}{2}}$ are, respectively, given by

$$
\begin{aligned}
\check{W}_{2 p+1}= & \sum_{n=2 p+1}^{\infty} n t_{n}\left[\sum_{k=1}^{\infty} k t_{k} \frac{\partial}{\partial t_{n+k-2 p-1}}+\frac{\hbar^{2}}{2} \sum_{k=0}^{n-2 p-1} \frac{\partial}{\partial t_{k}} \frac{\partial}{\partial t_{n-k-2 p-1}}\right. \\
& +\sum_{k=0}^{\infty}\left(\frac{n}{2}+k-p\right) \xi_{k+\frac{1}{2}} \frac{\partial}{\partial \xi_{k+n-2 p-\frac{1}{2}}}+\frac{\hbar^{2}}{2} \sum_{k=1}^{n-2 p-1} k \frac{\partial}{\partial \xi_{n-k-2 p-\frac{1}{2}}} \frac{\partial}{\partial \xi_{k-\frac{1}{2}}} \\
& \left.-\frac{\hbar}{2 \sqrt{\beta}}(1-\beta)(n-2 p) \frac{\partial}{\partial t_{n-2 p-1}}\right]+\sum_{n=2 p+1}^{\infty}\left(n+\frac{1}{2}\right) \xi_{n+\frac{1}{2}}\left[\sum_{k=1}^{\infty} k t_{k} \frac{\partial}{\partial \xi_{n+k-2 p-\frac{1}{2}}}\right. \\
& +\hbar^{2} \sum_{k=0}^{n-2 p-1} \frac{\partial}{\partial \xi_{k+\frac{1}{2}}} \frac{\partial}{\partial t_{n-k-2 p-1}}-\frac{\hbar}{\sqrt{\beta}}(1-\beta)(n-2 p) \frac{\partial}{\partial \xi_{n-2 p-\frac{1}{2}}} \\
& \left.+\sum_{k=0}^{\infty} \xi_{k+\frac{1}{2}} \frac{\partial}{\partial t_{k+n-2 p}}\right], \\
\check{W}_{k}= & a_{2 p+1-k} \sum_{n=2 p+1}^{\infty} n t_{n} \frac{\partial}{\partial t_{n-k}}+a_{2 p+1-k} \sum_{n=2 p+1}^{\infty}\left(n+\frac{1}{2}\right) \xi_{n+\frac{1}{2}} \frac{\partial}{\partial \xi_{n+\frac{1}{2}-k}}, \\
\check{W}_{l+\frac{1}{2}}= & \varepsilon_{2 p-l} \sum_{n=2 p+1}^{\infty} n\left(p-l+\frac{n}{2}\right) t_{n} \frac{\partial}{\partial \xi_{n-\frac{1}{2}-l}}+\varepsilon_{2 p-l} \sum_{n=2 p+1}^{\infty}\left(n+\frac{1}{2}\right) \xi_{n+\frac{1}{2}} \frac{\partial}{\partial t_{n-l}} . \quad(5.4)
\end{aligned}
$$

In similarity with the case of non-Gaussian supereigenvalue model (3.1), the partition function (5.1) can not be uniquely determined by the constraint (5.3). We have to consider the additional constraints. It is noted that the partition function (5.1) also satisfies the relations (3.10). Substituting (3.10) into (5.3), we obtain

$$
(D+\check{\mathcal{W}}) Z_{C N G}(t, \xi ; a, \varepsilon)=0,
$$

where $\check{\mathcal{W}}=\check{W}-\sum_{k=1}^{2 p} k^{2} t_{k} \frac{\partial}{\partial a_{k}}-\sum_{l=0}^{2 p}\left(l+\frac{1}{2}\right) \xi_{l+\frac{1}{2}} \frac{\partial}{\partial \varepsilon_{l}}$. 
Furthermore, from (5.5) we can derive

$$
Z_{C N G}(t, \xi ; a, \varepsilon)=\sum_{k=0}^{\infty}\left(-D^{-1} \check{\mathcal{W}}\right)^{k} e^{-\frac{\sqrt{\beta}}{\hbar} N t_{0}} Z_{C N G}(a, \varepsilon)
$$

where $Z_{C N G}(a, \varepsilon)$ is given by

$$
Z_{C N G}(a, \varepsilon)=\left(\prod_{i=1}^{N} \int_{0}^{+\infty} d z_{i} \int d \theta_{i}\right) \Delta_{N S}(z, \theta)^{\beta} e^{-\frac{\sqrt{\beta}}{\hbar} \sum_{i=1}^{N} \bar{V}_{N S}\left(z_{i}, \theta_{i}\right)} .
$$

Note that $\check{\mathcal{W}}$ in (5.6) contains noncommutative operators with degrees ranging from $\frac{1}{2}$ to $2 p+1$, it causes that the general expressions of the correlators (5.2) can not be obtained. In principle, we can calculate the correlators step by step from (5.6).

For example, we present the correlators (5.2) with $\sum_{\mu=1}^{n} k_{\mu}+\sum_{\nu=1}^{m} s_{\nu}+\frac{m}{2} \leq 3$. They are the same as (3.14) by replacing $Z_{N G}(a, \varepsilon)$ by $Z_{C N G}(a, \varepsilon)$, except for the case of $p=1$ in $(5.2)$

$$
\hat{C}_{3}(a, \varepsilon)=\frac{\hbar}{\sqrt{\beta}}\left[\frac{N \tilde{N}}{2}+\sum_{k=1}^{2} k a_{k} \frac{\partial}{\partial a_{k}}+\sum_{l=0}^{2}\left(l+\frac{1}{2}\right) \varepsilon_{l} \frac{\partial}{\partial \varepsilon_{l}}\right] Z_{C N G}(a, \varepsilon) .
$$

\section{Conclusions}

The $W$-representations of matrix models may realize the partition functions by acting on elementary functions with exponents of the given $W$ operators. They play an important role in the calculations of the correlators. For the supereigenvalue model in the Ramond sector, one has found its $W$-representation and derived the compact expressions of the correlators. In this paper, we have analyzed the Gaussian and chiral supereigenvalue models in the Neveu-Schwarz sector. Their partition functions (2.1) and (4.1) can be expressed as the infinite sums of the homogeneous operators $\left(-\tilde{D}^{-1} \tilde{W}\right)^{k}$ and $\left(-\tilde{D}^{-1} \bar{W}\right)^{k}$ acting on the function $e^{-\frac{\sqrt{\beta}}{\hbar} N t_{0}}$, i.e., (2.24) and (4.9), respectively. We noted that $\tilde{D}$ is not the degree operator. It leads to that the usual $W$-representations of these matrix models can not be provided here. In spite of this negative result, the notable feature has emerged from the studies of the correlators. Since the actions of the homogeneous operators $\left(-\tilde{D}^{-1} \tilde{W}\right)^{k}$ and $\left(-\tilde{D}^{-1} \bar{W}\right)^{k}$ on the function $e^{-\frac{\sqrt{\beta}}{\hbar} N t_{0}}$ can be evaluated explicitly, we have derived the compact expressions of the correlators (2.32) and (4.10) from (2.24) and (4.9), respectively. Our analysis provides additional insight into these supereigenvalue models.

We have also considered the non-Gaussian (chiral) supereigenvalue models in the Neveu-Schwarz sector. Unlike the cases of previous Gaussian and chiral supereigenvalue models, it was noted that the operators $\hat{\mathcal{W}}$ and $\check{\mathcal{W}}$ in (3.13) and (5.6) are not the homogeneous operators, which are constituted by the noncommutative operators with degrees ranging from $\frac{1}{2}$ to $2 p+2$ and $2 p+1$, respectively. This makes it quite difficult to derive the compact expressions of the correlators from (3.13) and (5.6).

Finally, it should be mentioned that the partition function for (deformed) Gaussian matrix models have the explicit character expansions [19]-[21], [33, 34, 41]. The character 
expansions imply that the expectation values of characters are again characters, which is considered as the superintegrability property of the models [42]. For the supereigenvalue models, whether there exists the character decomposition formulas deserves further study.

\section{Acknowledgments}

This work is supported by the National Natural Science Foundation of China (Nos. 11875194 and 11871350).

Open Access. This article is distributed under the terms of the Creative Commons Attribution License (CC-BY 4.0), which permits any use, distribution and reproduction in any medium, provided the original author(s) and source are credited.

\section{References}

[1] L. Álvarez-Gaumé, H. Itoyama, J.L. Manes and A. Zadra, Superloop equations and two-dimensional supergravity, Int. J. Mod. Phys. A 7 (1992) 5337 [hep-th/9112018] [INSPIRE].

[2] H. Itoyama, Integrable superhierarchy of discretized 2-D supergravity, Phys. Lett. B 299 (1993) 64 [hep-th/9206091] [INSPIRE].

[3] L. Álvarez-Gaumé, K. Becker, M. Becker, R. Emparan and J. Manes, Double scaling limit of the super-Virasoro constraints, Int. J. Mod. Phys. A 8 (1993) 2297 [hep-th/9207096] [INSPIRE].

[4] K. Becker and M. Becker, Nonperturbative solution of the super-Virasoro constraints, Mod. Phys. Lett. A 8 (1993) 1205 [hep-th/9301017] [INSPIRE].

[5] J.M. Figueroa-O'Farrill and S. Stanciu, On a new supersymmetric KdV hierarchy in 2-D quantum supergravity, Phys. Lett. B 316 (1993) 282 [hep-th/9302057] [INSPIRE].

[6] I.N. McArthur, The partition function for the supersymmetric Eigenvalue model, Mod. Phys. Lett. A 8 (1993) 3355 [INSPIRE].

[7] I.N. McArthur, A discrete integrable hierarchy related to the supersymmetric eigenvalue model, J. Phys. A 27 (1994) 4161 [inSPIRE].

[8] J.C. Plefka, Iterative solution of the supereigenvalue model, Nucl. Phys. B 444 (1995) 333 [hep-th/9501120] [INSPIRE].

[9] J.C. Plefka, The supereigenvalue model in the double scaling limit, Nucl. Phys. B 448 (1995) 355 [hep-th/9504089] [INSPIRE].

[10] G. Akemann and J.C. Plefka, The chiral supereigenvalue model, Mod. Phys. Lett. A 12 (1997) 1745 [hep-th/9705114] [INSPIRE].

[11] H. Itoyama and H. Kanno, Supereigenvalue model and Dijkgraaf-Vafa proposal, Phys. Lett. B 573 (2003) 227 [hep-th/0304184] [INSPIRE].

[12] P. Ciosmak, L. Hadasz, M. Manabe and P. Sułkowski, Super-quantum curves from super-eigenvalue models, JHEP 10 (2016) 044 [arXiv: 1608.02596] [INSPIRE].

[13] P. Ciosmak, L. Hadasz, M. Manabe and P. Sułkowski, Singular vector structure of quantum curves, arXiv:1711.08031 [INSPIRE]. 
[14] P. Ciosmak, L. Hadasz, Z. Jaskólski, M. Manabe and P. Sulkowski, From CFT to Ramond super-quantum curves, JHEP 05 (2018) 133 [arXiv: 1712.07354] [INSPIRE].

[15] V. Bouchard and K. Osuga, Supereigenvalue models and topological recursion, JHEP 04 (2018) 138 [arXiv: 1802.03536] [INSPIRE].

[16] V. Bouchard and K. Osuga, $\mathcal{N}=1$ super topological recursion, arXiv:2007.13186 [INSPIRE].

[17] K. Osuga, Topological recursion in the ramond sector, JHEP 10 (2019) 286 [arXiv: 1909.08551] [INSPIRE].

[18] A. Morozov and Sh. Shakirov, Generation of matrix models by $\hat{W}$-operators, JHEP 04 (2009) 064 [arXiv:0902.2627] [INSPIRE].

[19] A. Alexandrov, A. Mironov, A. Morozov and S. Natanzon, On KP-integrable Hurwitz functions, JHEP 11 (2014) 080 [arXiv:1405.1395] [INSPIRE]

[20] A. Morozov, On $W$-representations of $\beta$ - and $q, t$-deformed matrix models, Phys. Lett. $B \mathbf{7 9 2}$ (2019) 205 [arXiv: 1901.02811] [INSPIRE].

[21] H. Itoyama, A. Mironov and A. Morozov, Complete solution to Gaussian tensor model and its integrable properties, Phys. Lett. B 802 (2020) 135237 [arXiv:1910.03261] [INSPIRE].

[22] A. Alexandrov, Cut-and-Join operator representation for Kontsewich-Witten tau-function, Mod. Phys. Lett. A 26 (2011) 2193 [arXiv:1009.4887] [INSPIRE].

[23] A. Alexandrov, Cut-and-join description of generalized Brezin-Gross-Witten model, Adv. Theor. Math. Phys. 22 (2018) 1347 [arXiv:1608.01627] [INSPIRE].

[24] Y. Chen, R. Wang, K. Wu and W.-Z. Zhao, Correlators in the supereigenvalue model in the Ramond sector, Phys. Lett. B 807 (2020) 135563 [arXiv:2006.11013] [INSPIRE].

[25] J. Harer and D. Zagier, The Euler characteristic of the moduli space of curves, Invent. Math. 85 (1986) 457.

[26] C. Itzykson and J.B. Zuber, Matrix integration and combinatorics of modular groups, Commun. Math. Phys. 134 (1990) 197 [INSPIRE].

[27] B. Lass, Demonstration combinatoire de la formule de Harer-Zagier, C. R. Acad. Sci. Paris Ser. I 333 (2001) 155.

[28] S.K. Lando and A.K. Zvonkin, Graphs on surfaces and their applications, Springer Berlin Germany (2003).

[29] I.P. Goulden and A. Nica, A direct bijection for the Harer-Zagier formula, J. Comb. Theory A 111 (2005) 224.

[30] E. Akhmedov and Sh. Shakirov, Gluing of surfaces with polygonal boundaries, Funct. Anal. Appl. 43 (2009) 245 [arXiv:0712.2448].

[31] A. Morozov and Sh. Shakirov, From Brezin-Hikami to Harer-Zagier formulas for Gaussian correlators, arXiv: 1007.4100 [INSPIRE].

[32] A. Morozov and Sh. Shakirov, Exact 2-point function in Hermitian matrix model, JHEP 12 (2009) 003 [arXiv:0906.0036] [INSPIRE].

[33] H. Itoyama, A. Mironov and A. Morozov, Ward identities and combinatorics of rainbow tensor models, JHEP 06 (2017) 115 [arXiv:1704.08648] [INSPIRE].

[34] A. Mironov and A. Morozov, On the complete perturbative solution of one-matrix models, Phys. Lett. B 771 (2017) 503 [arXiv:1705.00976] [INSPIRE]. 
[35] B. Kang, K. Wu, Z.-W. Yan, J. Yang and W.-Z. Zhao, Exact correlators in the Gaussian Hermitian matrix model, Phys. Lett. B 798 (2019) 134986 [arXiv:1903.08310] [InSPIRE].

[36] A. Alexandrov, A. Mironov and A. Morozov, Partition functions of matrix models as the first special functions of string theory. 1. Finite size Hermitean one matrix model, Int. J. Mod. Phys. A 19 (2004) 4127 [hep-th/0310113] [INSPIRE].

[37] A. Alexandrov, A. Mironov and A. Morozov, Solving Virasoro constraints in matrix models, Fortsch. Phys. 53 (2005) 512 [hep-th/0412205] [INSPIRE].

[38] A. Alexandrov, A. Mironov and A. Morozov, Unified description of correlators in non-Gaussian phases of Hermitean matrix model, Int. J. Mod. Phys. A 21 (2006) 2481 [hep-th/0412099] [INSPIRE].

[39] C. Cordova, B. Heidenreich, A. Popolitov and Sh. Shakirov, Orbifolds and exact solutions of strongly-coupled matrix models, Commun. Math. Phys. 361 (2018) 1235 [arXiv:1611.03142] [INSPIRE].

[40] L. Cassia, R. Lodin and M. Zabzine, On matrix models and their q-deformations, JHEP 10 (2020) 126 [arXiv: 2007.10354] [inSPIRE].

[41] A. Morozov, A. Popolitov and Sh. Shakirov, On $(q, t)$-deformation of Gaussian matrix model, Phys. Lett. B $\mathbf{7 8 4}$ (2018) 342 [arXiv:1803.11401] [INSPIRE].

[42] A. Mironov and A. Morozov, Sum rules for characters from character-preservation property of matrix models, JHEP 08 (2018) 163 [arXiv:1807.02409] [INSPIRE]. 